\title{
Identification and analysis of Chrysanthemum nankingense NAC transcription factors and an expression analysis of OsNAC7 subfamily members
}

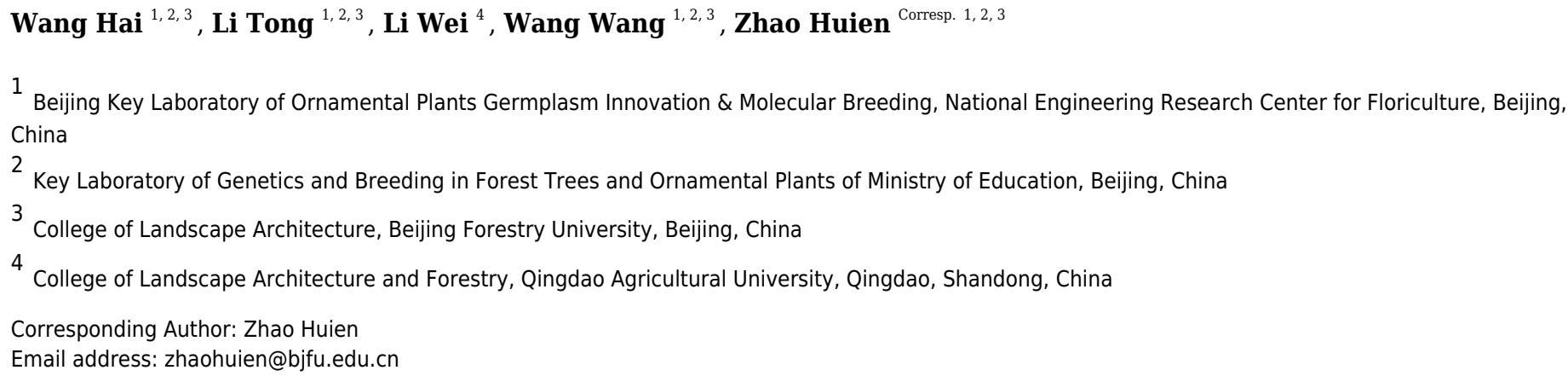

NAC (NAM, ATAF1-2, and CUC2) transcription factors (TFs) play a vital role in plant growth and development, as well as in plant response to biotic and abiotic stressors ${ }^{[1-2]}$. Chrysanthemum is a plant with strong stress resistance and adaptability; therefore, a systematic study of NAC TFs in chrysanthemum is of great significance for plant breeding. In this study, 153 putative NAC TFs were identified based on the Chrysanthemum nankingense genome. According to the NAC family in Arabidopsis and rice, a rootless phylogenetic tree was constructed, in which the 153 CnNAC TFs were divided into two groups and 19 subfamilies. Moreover, the expression levels of 12 CnNAC TFs belonging to the OsNAC7 subfamily were analyzed in C. nankingense under osmotic and salt stresses, and different tissues were tested during different growth periods. The results showed that these 12 OsNAC7 subfamily members were involved in the regulation of root and stem growth, as well as in the regulation of drought and salt stresses. Finally, we investigated the function of the CHR00069684 gene, and the results showed that CHR00069684 could confer improved salt and low temperature resistance, enhance ABA sensitivity, and lead to early flowering in tobacco. It was proved that members of the OsNAC7 subfamily have dual functions including the regulation of resistance and the mediation of plant growth and development. This study provides comprehensive information on analyzing the function of CnNAC TFs, and also reveals the important role of OsNAC7 subfamily genes in response to abiotic stress and the regulation of plant growth. These results provide new ideas for plant breeding to control stress resistance and growth simultaneously. 
1 Identification and analysis of Chrysanthemum nankingense

2 NAC transcription factors and an expression analysis of

3 OsNAC7 subfamily members

4

5

6

7

8

9

10

11

12

13

14

15

16

17

18

19

20

21

22

23

24

25

26

27

28

29

30

31

32

33

34

35

36

37

38

39

\author{
Hai Wang ${ }^{1,2,3}$, Tong $\mathrm{Li}^{1,2,3}$, Wei $\mathrm{Li}^{4}$, Wang Wang ${ }^{1,2,3}$, and Huien Zhao ${ }^{1,2,3}$ \\ 1. Beijing Key Laboratory of Ornamental Plants Germplasm Innovation \& Molecular Breeding; National Engineering Research Center for \\ Floriculture; \\ 2. Key Laboratory of Genetics and Breeding in Forest Trees and Ornamental Plants of Ministry of Education; \\ 3. College of Landscape Architecture, Beijing Forestry University, Beijing 100083, China; \\ 4. College of Landscape Architecture and Forestry, Qingdao Agricultural University, Qingdao, 266109, China.
}

Corresponding Author:

Huien Zhao ${ }^{1,2,3}$

No. 35 East Qinghua Road, Haidian District, Beijing, 100083, China

Email address: zhaohuien@bjfu.edu.cn

\begin{abstract}
NAC (NAM, ATAF1-2, and CUC2) transcription factors (TFs) play a vital role in plant growth and development, as well as in plant response to biotic and abiotic stressors ${ }^{[1-2]}$. Chrysanthemum is a plant with strong stress resistance and adaptability; therefore, a systematic study of NAC TFs in chrysanthemum is of great significance for plant breeding. In this study, 153 putative NAC TFs were identified based on the Chrysanthemum nankingense genome. According to the NAC family in Arabidopsis and rice, a rootless phylogenetic tree was constructed, in which the 153 CnNAC TFs were divided into two groups and 19 subfamilies. Moreover, the expression levels of 12 CnNAC TFs belonging to the OsNAC7 subfamily were analyzed in $C$. nankingense under osmotic and salt stresses, and different tissues were tested during different growth periods. The results showed that these 12 OsNAC7 subfamily members were involved in the regulation of root and stem growth, as well as in the regulation of drought and salt stresses. Finally, we investigated the function of the CHR00069684 gene, and the results showed that CHR00069684 could confer improved salt and low temperature resistance, enhance ABA sensitivity, and lead to early flowering in tobacco. It was proved that members of the OsNAC7 subfamily have dual functions including the regulation of resistance and the mediation of plant growth and development. This study provides comprehensive information on analyzing the function of CnNAC TFs, and also reveals the important role of OsNAC7 subfamily genes in response to abiotic stress and the regulation of plant growth. These results provide new ideas for plant breeding to control stress resistance and growth simultaneously.
\end{abstract}

\title{
Introduction
}


40 Transcription factors (TFs) are a class of important regulatory proteins, which inhibit or enhance gene expression by covalently binding to the DNA binding domain ${ }^{[3]}$. NAC (NAM, ATAF1-2, and CUC2) TFs are a large family endemic to plants and are rarely found in the genomes of bacteria, fungi, or animals ${ }^{[4]}$. NAC family proteins share a common NAC domain, which is identified by a consistent NAM sequence from Petunia and ATAF1/ATAF2 and CUC2 sequences from Arabidopsis. Arabidopsis ATAF is the first described member of NAC. NAM (NO APICAL MERISTEM) plays an important role determining the apical meristem and the location of primordia. Petunias with a $N A M$ gene mutation cannot form apical meristem ${ }^{[5]}$. The $C U C 2$ gene plays a role in the development of the embryo and flower, and mutations in CUC1 and $C U C 2$ cause the separation of cotyledons (embryonic organs), sepals, and stamens (floral organs), leading to defects in the formation of terminal bud meristematic tissues ${ }^{[6-8]}$. NAC family genes have about 160 residues at the N-terminus, forming a typical highly conserved domain, which is divided into five subdomains. Among them, three subdomains are highly conserved, and two subdomains are diverse. These two subdomains are related to the multi-functions of the NAC family $\mathrm{TFs}^{[1,9]}$.

NAC TFs are expressed during different development stages and in different tissues of plants, which are closely related to plant growth and development, as well as the regulation of plant response to abiotic and biotic stresses ${ }^{[10]}$. Ooka et al. comprehensively analyzed 75 predicted NAC TFs in rice (Oryza sativa) and 105 predicted NAC TFs in Arabidopsis (Arabidopsis thaliana) and divided the NAC TFs into two groups (Group 1 and Group 2) according to similarities in their structural domains. Group 1 contains 14 subfamilies and Group 2 contains four subfamilies ${ }^{[9]}$. Rushton et al. analyzed the evolutionary relationships among 450 NAC proteins in rice (O. sativa), Arabidopsis (A. thaliana), tobacco (Nicotiana tabacum), poplar (Populus trichocarpa), and other plants, and divided the NAC gene family into seven subfamilies. Among them, six subfamilies exist in all plants, while the other subfamily only occurs in tobacco, pepper, potato, and tomato ${ }^{[11]}$. So far, 151 NAC family members have been found in O. sativa ${ }^{[4]}, 117$ in A. thaliana ${ }^{[4,9]}, 152$ in tobacco $(N \text {. tabacum })^{[11]}, 205$ in soybean

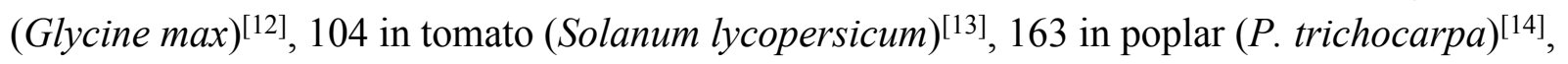
79 in grape (Vitis vinifera) ${ }^{[15]}, 168$ in durum wheat (Triticum turgidum $)^{[16]}$, and 488 in bread wheat (Triticum aestivum) $)^{[2]}$.

Studies on NAC TFs have reported that the NAC genes respond to at least one biotic or abiotic stress, such as saline-alkali, drought, cold, or exogenous abscisic acid (ABA), which play important roles in plant drought tolerance, saline-alkali tolerance, virus resistance, and the cold resistance response ${ }^{[15,17]}$. In previous studies, most NAC TFs are closely related to salt and alkali tolerance, cold tolerance, and disease resistance of plants, whereas other NAC TFs improve plant resistance to multiple stressors at the same time ${ }^{[18-20]}$. The $A$. thaliana NAC TFs ATAF1, ANAC016, ANAC019, ANAC055, and ANAC072[21-22]; the O. sativa NAC TFs OsNAC045, OsNAC10, and OsNAC022 [23]; the G. $\max$ NAC TFs GmNAC085, GmNAC109, GmNAC30, GmNAC81, GmNAC003, GmNAC004, GmNAC11, and GmNAC20[24-26]; and the T. turgidum NAC TF TaRNAC1 ${ }^{[26-27]}$ enhance drought tolerance. The Lilium regale NAC TF LrNAC35, the 
80

81

82

83

84

85

86

87

88

89

90

91

92

93

94

95

96

97

98

99

100

101

102

103

104

105

106

107

108

109

110

111

112

113

114

115

116

117

118

119

S. lycopersicum NAC TFs SINAC1 and SINAC61, and the A. thaliana NAC TFs ATAF2 and TIP are all related to virus resistance in plants ${ }^{[18,28-31]}$. The G. $\max$ NAC TF GmNAC20 significantly improves cold resistance in plants ${ }^{[19,26,32]}$. The $O$. sativa NAC TFs OsNAC5 and OsNAC6 (the ATAF subfamily) improve drought resistance, salt tolerance, and freeze resistance of transgenic plants ${ }^{[33-34]}$ and the $V$. vinifera NAC TF VvNAC17 enhances the sensitivity of $A$. thaliana to ABA and improves salt tolerance, cold tolerance, and drought tolerance ${ }^{[15]}$.

Most genera in Anthemideae are distributed in southern Africa and the Mediterranean region, but are also widely distributed in Europe and Asia. The main economic crops in the family include Chrysanthemum carinatum Schousb., C. segetum L., C. nankingense, Dendranthema morifolium (Ramat.) Tzvel., Pyrethrum cinerariifolium Trev., and Artemisia annua. These are important vegetables, ornamental flowers, grasses, and herbs. Although Anthemideae plants are widely distributed, only some of the currently available plants can be cultivated in arid, saline, or cold regions, as their various traits are weakened and can only be planted as single-season cultivars. Under adverse circumstances, they lose the advantages of being an easy-to-manage perennial, leading to difficulties in popularity and application. Improving the growth characteristics and resistance to drought and saline-alkali stresses of Anthemideae plants would significantly improve their application range and enhance their cultural advantages. NAC TFs play an important role in abiotic stress in plants ${ }^{[19]}$. Therefore, regulating the CnNAC TFs is an effective way to enhance the resistance of Anthemideae plants. However, most studies on the Chrysanthemum NAC genes are single-gene studies that focus on disease and insect resistance. Few comprehensive studies have been performed on CnNAC TFs and their abiotic stress responses. In this study, CnNAC TFs were analyzed based on published $C$. nankingense genome data. In addition, the expression levels of the CnNAC TFs belonging to the OsNAC7 subfamily were analyzed in different tissues at different growth stages in C. nankingense under osmotic and salt stresses. These results are expected to contribute to resistance breeding and growth and developmental regulation of Chrysanthemum.

\section{Materials \& Methods}

\section{Gene retrieval of $C$. nankingense NAC TFs}

The $A$. thaliana NAC TF sequences were obtained from the Arabidopsis Information Resource (http://www.arabidopsis.org/). C. nankingense genomic data were downloaded from the website of the Institute of Chinese Medicine, Chinese Academy of Chinese Medical Sciences (http://www.amwayabrc.com/zh-cn/download.htm). Based on the $A$. thaliana NAC protein sequences, HMMER3.0 software was used to construct a multiple comparison HMM model, which was then used to search the $C$. nankingense genome protein data to obtain the CnNAC protein sequences. The CnNAC protein sequences were identified and screened using the SMART tool (http://smart.embl-heidelberg.de/) and Pfam (https://pfam.xfam.org/), and the predicted CnNAC TF members were obtained. The CnNAC TFs were used for subsequent analyses based on this comprehensive screening ${ }^{[35]}$.

\section{Bioinformatics analysis of $C$. nankingense NAC TFs}


120 The NAC TFs of $C$. nankingense, A. thaliana, and some of those of $O$. sativa were used to

121

122

123

124

125

126

127

128

129

130

131

132

133

134

135

136

137

138

139

140

141

142

143

144

145

146

147

148

149

150

151

152

153

154

155

156

157

158

159

construct a rootless phylogenetic tree using the multiple comparison function in ClustalW (default parameters) and the neighbor-joining (NJ) method in MEGA-X software according to the existing $A$. thaliana and $O$. sativa NAC gene family classification system ${ }^{[1,9,36]}$. The rootless phylogenetic tree was used to study the phylogenetic relationships of the CnNAC TFs ${ }^{[37]}$. MEME online software (http://meme-suite.org/tools/meme) was used to predict the conserved motifs of the CnNAC TFs. The ProtParam online tool (https://web.expasy.org/protparam/) was used to analyze the physical and chemical properties of the CnNAC TF amino acid sequences and predict their subcellular locations ${ }^{[38]}$. The TMHMM online tool (http://www.cbs.dtu.dk/services/TMHMM-2.0/) was used to predict the protein transmembrane structure of the CnNAC TFs. The three-dimension (3D) models of the conserved NAC domains of the CnNAC proteins were predicted by Phyre2

(http://www.sbg.bio.ic.ac.uk/phyre2/html/page.cgi?id=index ${ }^{[39]}$.

\section{Growth conditions and treatment of the plant materials}

C. nankingense seeds (from Beijing Forestry University) were sown on peat to produce seedlings under growing conditions of $25^{\circ} \mathrm{C} / 23^{\circ} \mathrm{C}$ (day/night), $16 \mathrm{~h} / 8 \mathrm{~h}$ (day/night), and 2,000 Lux. The roots, stems, and 3-5 new leaves on the top of the seedlings were collected and quickly flash frozen in liquid nitrogen as experimental materials at 28,44 , and $60 \mathrm{~d}$ after seeding, respectively, and stored at $-80^{\circ} \mathrm{C}$. Three replicates were taken for each period.

Rooted C. nankingense seedlings obtained from stem hydroponics were subjected to osmotic and salt stresses. The side shoots of $C$. nankingense seedlings grown for $44 \mathrm{~d}$ were cut out and inserted into cultivation medium containing MS medium and $0.1 \mathrm{mg} / \mathrm{L}$ IBA. The growing conditions were $25^{\circ} \mathrm{C} / 23^{\circ} \mathrm{C}$ (day/night), $16 \mathrm{~h} / 8 \mathrm{~h}$ (day/night), and 2,000 Lux. The cultivation medium was changed every $3-5 \mathrm{~d}$, and rooted seedlings were obtained after $20 \mathrm{~d}$. Then, the cultivation solution was changed to $20 \%$ PEG6000 or $200 \mathrm{mM} \mathrm{NaCl}$ solution for the osmotic and salt stress treatments, respectively. Samples were taken at $0,1,4,8,12,24$, and $36 \mathrm{~h}^{[40]}$. The entire rooted seedling was taken as a sample and stored at $-80^{\circ} \mathrm{C}$ after flash-freezing in liquid nitrogen. Three replicates were taken for each period.

\section{RNA isolation and real-time quantitative-polymerase chain reaction (RT-qPCR) analysis} Total RNA was isolated using the FastPure Plant Total RNA Isolation Kit (Code No. RC401-01; Nanjing Vazyme Biotechnical Co., Ltd., Nanjing, China). The reverse transcription of RNA was carried out using the PrimeScript ${ }^{\mathrm{TM}}$ RT reagent Kit with the gDNA Eraser (Perfect Real Time, Code No. RR047A; Takara Bio., Shiga, Japan). The primers used to analyze the expression of the OsNAC7 subfamily members were designed based on the C. nankingense genome (Table 1). RT-qPCR was carried out with on the Applied Biosystems StepONEPlus Real Time PCR System (Foster City, CA, USA), using ChamQ Universal SYBR qPCR Master Mix (Code No. Q711-03; Nanjing Vazyme Biotechnical Co., Ltd., Nanjing, China). The reaction system was 40 cycles of $95^{\circ} \mathrm{C}$ for $20 \mathrm{~s}, 95^{\circ} \mathrm{C}$ for $3 \mathrm{~s}, 60^{\circ} \mathrm{C}$ for $30 \mathrm{~s}, 95^{\circ} \mathrm{C}$ for $15 \mathrm{~s}$, and $60^{\circ} \mathrm{C}$ for $1 \mathrm{~min}^{[42]}$, with actin 7 from C. nankingense as the internal reference gene ${ }^{[41]}$. The relative expression of genes was calculated by the $2^{-\Delta \mathrm{Ct}}$ method.

Peer) reviewing PDF | (2021:01:57609:2:0:NEW 20 Apr 2021) 
160

161

162

163

164

165

166

167

168

169

170

171

172

173

174

175

176

177

178

179

180

181

182

183

184

185

186

187

188

189

190

191

192

193

194

195

196

197

198

199

\section{Subcellular localization and functional analysis}

The super 1300 vector was used as the overexpression vector (kindly provided by Professor Huitang Pan of Beijing Forestry University, Beijing, China. Supplementary Data 1). The vector uses super35S as the promoter. According to the open reading frame (ORF) of the CHR00069684 gene (Supplementary Data 2) and the polyclonal sites of the super1300 vector, the endonucleases Pst I and Kpn I (NEB, Beijing) were selected to construct the binary expression vector according to the double enzyme method. The recombinant plasmid was transformed into Escherichia coli competent cells (DH5 $\alpha$ ), which were inoculated on solid LB medium containing $50 \mathrm{mg} / \mathrm{L}$ kanamycin; the resistance $E$. coli cells were validated by colony PCR. The obtained positive plaques were sent to the sequencing company (Beijing Ruibio BiotechCo.,Ltd) for sequencing and plasmid recovery. The super35S::GFP and super35S::CHR00069684::GFP vectors were introduced into Agrobacterium tumefaciens strain GV3101 (Weidi Biotechnology, Shanghai, China) by the freeze-thaw method and then inoculated on LB medium supplemented with $50 \mathrm{mg} / \mathrm{L}$ kanamycin, $50 \mathrm{mg} / \mathrm{L}$ gentamicin, and 50 $\mathrm{mg} / \mathrm{L}$ rifampicin. The surviving plaques were validated by colony $\mathrm{PCR}$, and the positive plaques were inoculated into LB liquid medium containing $50 \mathrm{mg} / \mathrm{L}$ kanamycin, $50 \mathrm{mg} / \mathrm{L}$ gentamicin, and $50 \mathrm{mg} / \mathrm{L}$ rifampicin for propagation. The transgenic tobacco (NT78) was obtained through $A$. tumefaciens-mediated transformation ${ }^{[43]}$, screened by $30 \mathrm{mg} / \mathrm{L}$ hygromycin, and validated by PCR identification of the target gene. CHR000696884::GFP fusion protein and GFP protein signals were observed by a laser confocal microscopy (Leica TCS SP5) in roots, stems, and leaves of the transgenic tobacco plants. The super35S::GFP vector was used as control.

\section{Results}

\section{Identification of the CnNAC TFs and the phylogenetic analysis}

According to the $A$. thaliana NAC protein sequences, 153 predicted CnNAC TFs were identified from the $C$. nankingense genome data using HMMER3.0. Using ClustalW in MEGA, $153 C$. nankingense NAC proteins, 105 A. thaliana NAC proteins, and 6 O. sativa NAC proteins were multi-sequence aligned, and a rootless phylogenetic tree was constructed using the $\mathrm{NJ}$ method by MEGA (Fig. 1, Supplementary Data 3). According to the existing A. thaliana and O. sativa NAC gene family classification system ${ }^{[1,9,36]}$ and the topology of the rootless phylogenetic tree, the CnNAC TFs were divided into two groups of 19 subfamilies, including 17 known subfamilies and two unknown subfamilies. Group 1 contained 15 subfamilies, including OsNAC7, NAC1, NAM, ANAC077, ANAC011, OsNAC8, TIP, NAC2, ONAC022, TERN, SENU5, NAP, AtNAC3, ATAF, and unclassified 2, with a total of 111 members. Group 2 contained five subfamilies, which were ANAC063, ONAC003, ANAC007, unclassified 1, and ONAC001, with a total of 42 members. Among them, the ANAC077 subfamily occurred in both groups. The different branches of the phylogenetic tree testify to the functional diversity of this family. According to further analysis of the CnNAC TFs (Supplementary Fig. 1), 104 NAC genes were detected in the 17 known subfamilies, and $49 N A C$ genes were detected in the two unknown subfamilies. Three known subfamilies (ONAC022, NAC2, and OsNAC7) contained 13, 11, and 
20012 members respectively, with the greatest numbers of genes among all known subfamilies.

201 However, the two unknown subfamilies, unclassified 1 and unclassified 2, contained 28 and 21

$202 N A C$ genes, respectively.

203 Prediction of the protein structure is considered as a reliable analytical method to understand the 204 molecular function of proteins ${ }^{[39]}$. Therefore, the 3D models of one gene from each branch of the 205 NAC phylogenetic trees (Fig. 1), and all 60 CnNAC family members were constructed using the 206 Phyre2 server (Fig. 2). c3ulxA ${ }^{[44]}$ and d1 ut7 $\mathrm{a}^{[45]}$ were used as the reference model template, and 207 the confidence level was set as $100 \%$. The c3ulxA template is a DNA binding protein and 208 annotated as stress-induced NAC1 according to the crystal structure of the conserved domain of 209 rice stress responsive NAC1 in the PDB database. The d1ut7a template is annotated as a NAC 210 domain. The 3D modeling results revealed that these CnNAC TFs possessed similar tertiary 211 structures, implying that CnNAC TFs may evolved from the same ancestor sequence and/or 212 under purifying selection to maintain stabilization during the long-term evolution after the initial 213 divergence ${ }^{[46]}$.

214 Analysis of the CnNAC TF conserved motifs

215 The online tool MEME-Motif Suite was used to analyze the motifs of the 153 predicted CnNAC 216 TFs (Fig. 3) and the positions of the 15 motifs on different genes were determined (Fig. 4). As a 217 result (Supplementary Fig. 2), five genes (CHR00045488-RA, CHR00007510-RA, 218 CHR00036356-RA, CHR00079376-RA, and CHR00089260-RA) containing 11 motifs, 219 respectively, constituted the most abundant motifs. These five genes all belonged to the unknown 220 unclassified 1 subfamily. Nine genes (CHR00007511-RA, CHR00035414-RA, CHR00039887221 RA, CHR00039888-RA, CHR00055754-RA, CHR00055752-RA, CHR00088453-RA, 222 CHR00024060-RA, and CHR00058504-RA) containing 10 motifs, respectively, were also in the 223 unknown subfamilies (six in unclassified 1 and three in unclassified 2). Sixty-two genes, which 224 contained the highest number of 7 motifs, were distributed in 12 known subfamilies and two 225 unknown subfamilies.

226 According to the comparative analysis of the motifs in different subfamilies (Fig. 4), the included 227 motif bases were in motifs 1-7 in the known subfamilies of Group 1, but different subfamilies 228 contained different numbers of motifs and different conserved motifs. For example, motifs 4, 6, 2293 , and 7 were in the NAM subfamily and motifs 1, 4, 2, 5, and 6 were in the OsNAC7 subfamily. 230 However, some members in the unknown subfamily unclassified 2 had more motifs, such as 231 motifs 10, 13, and 14, than in the other subfamilies. Each member in the known subfamilies of 232 Group 2 contained far fewer motifs than those in the known subfamilies of Group 1. Members in 233 the known subfamilies of Group 2 contained motifs 1, 3, 4, 5, 6, and 7, and lacked motif 2 234 compared to those in the known subfamilies of Group 1. However, the CnNAC TFs in 235 unclassified 1 of Group 2 had the highest number of motifs, most of which were motifs 7-11.

236 Subcellular localization, protein transmembrane analysis, and physicochemical property 237 analysis of CnNAC TFs

238 According to the subcellular localization and transmembrane analysis results (Supplementary

239 Table 1), 106 of the 153 CnNAC TFs were located in the nucleus and 47 were located outside 
240 the cell. Of the 111 members in Group 1, 106 were nuclear and 15 were extracellular, of which 241 the 21 members within the unclassified 2 subfamily were all nuclear. Only 10 of the 42 members 242 of Group 2 were located in the nucleus, the other 32 were located outside the cell, and 23 of the 24328 members of the unclassified 1 subfamily of Group 2 were located outside the cell. However, 244 the protein transmembrane analysis of the 153 NAC TFs revealed that only one member of 245 Group 1 was expressed inside the membrane and eight members were expressed transmembrane, 246 while the remaining 102 members were all expressed outside the membrane. There were no 247 transmembrane-expressed Group 2 members, while two members were expressed inside the 248 membrane and 40 members were expressed outside the membrane. The three genes expressed 249 inside the membrane were all located in the nucleus, and the genes expressed transmembrane and 250 outside the membrane all had members located in the nucleus and extracellularly, respectively. 251 The physicochemical properties of the CnNAC TFs were analyzed (Supplementary Table 2). The 252 lengths of the CnNAC TF amino acids ranged from 68 to 693 (mean length = 307.99). The 253 254 255 256 257 258 259

260

261

262

263

264 molecular weight range of the CnNAC TFs was 7,787.81-80,985.3 Da with an average molecular weight of 35,195.1 Da. The theoretical isoelectric point (pI) range of the CnNAC TFs was $4.43-10.2$, and the average $\mathrm{pI}$ was 7.09 . The grand average of hydropathy was -0.72896 , while the lowest value was -1.37 , and the highest value was -0.275 . The aliphatic amino acid index was 44.15-94.84, with a mean value of 64.58 .

\section{Analysis of the OsNAC7 subfamily gene expression levels during different $C$. nankingense} growth stages

The OsNAC7 subfamily included 12 genes in A. thaliana, including SND1, NST1, URP7, $B R n 1 / 2$, and $V N D 1 / 2 / 3 / 4 / 5 / 6 / 7$, the functions of which are all related to regulation of the formation of secondary cell walls in plant stems, roots, and anthers. Here, we analyzed the expression levels of the OsNAC7 subfamily genes in the roots, stems, and leaves of $C$. nankingense during different growth stages (Figs. 5 and 6, Supplementary Table 3).

265

266 As a results, all the 12 members of the $C$. nankingense OsNAC7 subfamily played a role in roots, stems, and leaves. The expression levels of most of the OsNAC7 genes were generally low in the roots and leaves but high in the stems. However, differences in the expression levels were observed during different root, stem, and leaf developmental stages. Ten out of the 12 members in the OsNAC7 subfamily were expressed at higher levels in the stems at $44 \mathrm{~d}$, including CHR00068684, CHR00042500, CHR00009966, CHR00043953, CHR00073261, CHR00032377, CHR00026420, CHR00003673, CHR00027311, and CHR00043175. CHR00048081 was

272

273 expressed at high levels in the stems at $60 \mathrm{~d}$. CHR00020838 was expressed at high levels in the stems during the whole growth process, which were much higher than those in roots and leaves. Five out of the 12 members in the OsNAC7 subfamily were significantly related to root growth.

276

277 Among the 12 members. CHR00048081-RA was expressed at a higher level at $28 \mathrm{~d}$, CHR00020838-RA and CHR00043175-RA had higher levels at $44 \mathrm{~d}$, and CHR00009966-RA and

278 $C H R 00073261-R A$ had higher levels at $44 \mathrm{~d}$ and $60 \mathrm{~d}$. Five out of the 12 genes were expressed 279 at higher levels in leaves at $60 \mathrm{~d}$, such as CHR00043953-RA, CHR00032377-RA, CHR00026420RA, CHR00003673-RA, and CHR00043175-RA. 
280 Expression analysis of the $C$. nankingense OsNAC7 subfamily genes under the osmotic and 281 salt stress treatments

282 All $A$. thaliana OsNAC7 subfamily genes are associated with secondary metabolism ${ }^{[47]}$.

283 However, among the $O$. sativa OsNAC7 subfamily genes, ONAC106 inhibits leaf senescence

284 and also increases salt damage and tillering angle ${ }^{[3,48]}$; the expression levels of $O N A C 052$,

$285 O N A C 056$, and ONAC084 are downregulated under high salt and drought conditions ${ }^{[49]}$. Here,

286 we analyzed the expression levels of the $C$. nankingense OsNAC7 subfamily genes under

287 osmotic and salt stresses (Fig. 7, Fig. 8, Supplementary Table 3).

288 The results of gene expression under osmotic stress (Fig. 7) revealed that all of the 12 genes in

289 the OsNAC7 subfamily were regulated by osmotic stress, but their expression patterns were not

290 consistent. Among them, the expression levels of CHR00003673-RA, CHR00027311-RA, and

$291 C H R 00043175-R A$ continued to increase after treatment, indicating that these three genes may

292 play a key role improving the resistance to osmotic stress in C. nankingense. The CHR00026420-

$293 R A$ expression fluctuated but was always higher than that of the control group, indicating that

294 this gene is expressed during osmotic stress. CHR00069684-RA showed an obvious response to

295 osmotic stress. The expression of $C H R 00009966-R A$ and $C H R 00048081-R A$ was inhibited at the

296 initial stage of stress, and then gradually returned to normal expression levels. The response of

297 CHR00042500-RA and CHR00020838-RA was not obvious, and CHR00043953-RA and

$298 C H R 00073261-R A$ were severely inhibited by osmotic stress.

299 The gene expression results under salt stress (Fig. 8) revealed that all of the 12 genes in the

300 OsNAC7 subfamily responded to salt stress, and were initially upregulated and then

301 downregulated or were first downregulated and then upregulated; all decreased to the expression

302

level of those of the control group or below by $36 \mathrm{~h}$. The expression levels of CHR00069684-RA,

303 CHR00009966-RA, CHR00048081-RA, CHR00043953-RA, CHR00026420-RA, CHR00003673-

304 $R A, C H R 00027311-R A$, and $C H R 00043175-R A$ increased first at different time points and then

305 decreased. The significantly upregulated expression levels of these eight genes indicate that they

306 may be important in the salt stress response. CHR00042500-RA, CHR00020838-RA,

307

308 $C H R 00073261-R A$, and $C H R 00032377-R A$ were slightly inhibited by salt stress, although their

309 initial responses to salt stress were not obvious.

310 Subcellular localization and functional analysis

311

In this study, $C H R 00069684$ that was closely related to the NST protein was used to construct

312 the super35S::CHR00069684::GFP vector, which was then transformed into large leaf tobacco for subcellular localization observation and functional validation. GFP signals were observed in

313 the nucleus and cell membrane of leaves, stems, and roots of super35S::GFP transformed

314 tobacco plants (Fig. 9). No fluorescence signal was detected in the

315 super35S::CHR00069684::GFP transformed tobacco leaves (Supplementary Figure 3), whereas 316 there were strong GFP signals in the cell membrane of their roots and stems (Fig 9 E F). The 317 reason why the $35 \mathrm{~S}$ promoter-guided CHR00069684 expression showed tissue specificity need 318 to be further studied in the future. 
319 The super35S::CHR00069684::GFP transformed and control tobacco plants were treated with

320

321

322

323

324

325

326

327

328

329

330

331

332

333

334

335

336

337

338

339

340

341

342

343

344

345

346

347

348

349

350

351

352

353

354

355

356

357

$200 \mathrm{mM} \mathrm{NaCl}, 200 \mathrm{mM} \mathrm{ABA}$, or a low temperature of $4{ }^{\circ} \mathrm{C}$ (Fig. 10). The results showed that the transgenic tobacco plants were more resistant to salt and low temperature and more sensitive to ABA than the control plants. Moreover, the differences in the growth and development of tobacco plants were observed (Fig. 11); the flowering time of the transgenic tobacco was $10 \mathrm{~d}$ earlier than that of the control plants.

\section{Discussion}

According to the phylogenetic tree (Fig. 1) and motif analyses (Fig. 4) of CnNAC TFs, we found that the distinct NAC TF subfamilies each containing the most closely related members exhibited highly similar motif components. This included members in the OsNAC7 subfamily (Fig. 12), all of which possess motifs 1-6. The members clustering with ANAC012, ANAC066, ANAC043 ${ }^{[50-}$ 52], ANAC033, ANAC070, and ANAC015[53-54], which were annotated as NST1-NST3, URP7, and $B R N 1 / 2$, respectively, have the same motifs $1-7$, and the remaining part was clustered with $V N D 1-V N D 7^{[55-58]}$, which lack the motif 6 or motif 7 (Fig. 4). These results indicate that the CnNAC TFs in the same subfamily have similar motifs and similar functions ${ }^{[50-58]}$.

The NAC TFs are involved in various stages of plant growth and development and the responses to various stressors ${ }^{[25]}$. It was demonstrated in $O$. sativa, A. thaliana, and Asteraceae (Table 2) that most NAC TFs regulate plant growth, secondary metabolism, and growth and development time by changing root or stem growth, and further regulate the resistance of plants to biotic and abiotic stressors, but each subfamily had specific characteristics ${ }^{[25]}$. Compared to studies of Chrysanthemum and O. sativa, the ATAF subfamily member AaNACl ( $N A C 1$ in A. annua, KX082975.1) ${ }^{[59]}$, which was similar to CHR00002022-RA, CHR00045168-RA, and DgNAC1 (NACl in D. grandiform ${ }^{[60]}$, has the same function as the $O$. sativa ATAF subfamily genes OsNAC5 and OsNAC6 $6^{[33,61-63]}$. All of these genes improve drought resistance, salt tolerance, and low temperature tolerance of plants. The ONAC022 subfamily member DlNACl ( NACl in D. nankingense) ${ }^{[59]}$, which was similar to CHR00056910-RA and $O S N A C 063^{[59,64-65]}$, is involved in regulating drought-tolerance and salt-tolerance in plants. The functions of members belonging to the ATAF and ONAC022 subfamilies in chrysanthemum and rice are essentially the same, suggesting that closely related genes often have similar functions, so analyses of phylogenetic trees can be used to predict the functions of genes in the same subfamily. Through the $N A C$ gene functions of $O$. sativa and A. thaliana, it has been speculated that the CnNAC genes in the NACl subfamily may improve the overall stress resistance of plants ${ }^{[66]}$. The NAM subfamily genes improve tolerance to drought and high salt stress, which affect plant height and flowering time, and accelerate leaf senescence ${ }^{[67-70]}$. The NAP subfamily genes enhance drought tolerance and salt tolerance in plants by influencing the size of the epidermis, cortex, and stellate cells in roots [71-72]. The ONAC001 subfamily genes are induced by salt and drought stresses ${ }^{[73-74]}$, the TERN subfamily genes may be associated with disease resistance ${ }^{[75]}$, and the SENU5 subfamily genes enhance plant tolerance to salt, alkali, and drought stress ${ }^{[76-77]}$. 
358

359

360

361

362

363

364

365

366

367

368

369

370

371

372

373

374

375

376

377

378

379

380

381

382

383

384

385

386

387

388

389

390

391

392

393

394

395

396

397

Moreover, the two unknown subfamilies in CnNAC TFs (i.e., unclassified 1 and unclassified 2) contained more genes than the other subfamilies and had the most abundant numbers of motifs among the CnNAC TFs, indicating that these two unknown subfamilies may have diverse functions and may be involved in a wider range of growth and stress regulatory functions. The diversity of NAC family gene functions may be the reason why chrysanthemums, as a young and highly evolved large family, have strong adaptability to changing environments. According to the subcellular localization and transmembrane analysis results (Supplementary Table 1), most CnNAC TFs were related to nuclear genes; however, most of them were mainly expressed outside the membrane. For example, CHR00069684 was a nuclear gene, but was expressed outside the membrane (Supplementary Table 1); CHR00069684 was expressed in the cell membrane of tobacco, and could only be observed in the stems and roots of transgenic tobacco plants (Fig. 9). These results are consistent with the finding that TFs found in poplar can be localized to the nucleus and cytoplasm at the same time, but they are transferred to and expressed in the nucleus when regulated by an external stress signa ${ }^{[78]}$.

According to the phylogenetic tree (Fig. 1), the C. nankingense OsNAC7 subfamily members, including CHR00069684-RA, CHR00042500-RA, CHR00020838-RA, and CHR00009966-RA, were annotated as NST1-NST3 and have higher expression levels in the $C$. nankingense stems at $44 \mathrm{~d}$ (Fig. 6), which showed similar functions to the NST protein in A. thaliana ${ }^{[50-52,79-80]}$. Moreover, CHR00009966-RA likely played a more important role in the root growth. CHR00048081-RA, CHR00043953-RA, and CHR00073261-RA were annotated as URP7, BRN2, and $B R N 1$ respectively, which are mainly responsible for the root cap. The expression of these three genes in the C. nankingense roots was similar to that of the URP7 and BRN1/2 genes in $A$. thaliana $^{[53-54]}$. However, the expression of these three genes was higher in stems than in roots, indicating that $C H R 00048081-R A$ might play a regulatory role in the root growth and aging of the stem; CHR00043953-RA might play a regulatory role in the rapid growth of the stem and aging of the leaf; and $C H R 00073261-R A$ might play a regulatory role in the rapid growth of the stem. CHR00032377-RA, CHR00026420-RA, CHR00003673-RA, CHR00027311-RA, and CHR00043175-RA corresponded to the VND1,VND2, VND3, VND4, VND5, VND6, and VND7 genes, which co-participate in regulating the thickening of the secondary cell walls in $A$. thaliana [55-58, 81]. These genes were all involved in the stem growth and the aging process of leaves at the later growth stages (Fig. 6). Besides, CHR00043175-RA was also involved in the root growth. All genes responded to the osmotic (Fig. 7) and salt stresses (Fig. 8), which has only been studied in the ONAC106 gene of the OsNAC7 subfamily in rice ${ }^{[3,48]}$. Among them, CHR00026420-RA, CHR00003673-RA, CHR00027311-RA, and CHR00043175-RA may play a key role improving the resistance to osmotic stress in chrysanthemum. CHR00069684-RA, CHR00009966-RA, CHR00048081-RA, CHR00043953-RA, CHR00026420-RA, and CHR00003673-RA may be important genes in the salt stress response. To test this hypothesis, the super35S::CHR00069684::GFP transgenic tobacco lines were treated with salt stress, and the results confirmed the active function of CHR00069684 in the regulation of salt stress. In addition, the results obtained from $\mathrm{ABA}$ and low temperature treatments of the 
398

399

400

401

402

403

404

405

406

407

408

409

410

411

412

413

414

415

416

417

418

419

420

421

422

423

424

425

426

427

428

429

430

431

432

433

434

435

436

437

438

439

440

441

442

super35S::CHR00069684::GFP transgenic tobacco plants indicated that CHR00069684 enhanced ABA sensitivity and low temperature stress resistance in the transgenic tobacco plants, leading to reduced growth potential and premature flowering. Functional analysis on CHR00069684 confirmed the dual roles of the OsNAC7 subfamily genes in the growth and stress regulation of plants. However, the function of the OsNAC7 subfamily members during abiotic stress remains speculative, and many other functions of the OsNAC7 subfamily genes remain to be further explored.

\section{Conclusions}

In this study, the CnNAC TFs were divided into two groups with 19 subfamilies according to the phylogenetic tree. These 19 subfamilies consisted of 17 known subfamilies and two unknown subfamilies. The conserved motifs, subcellular localization, transmembrane localization, and physicochemical properties of the CnNAC TFs were comprehensively analyzed. Combined with research on the NAC family genes of A. thaliana, O. sativa, and Asteraceae, the functions of the CnNAC TFs were investigated. Analyses on the expression of the $12 C n N A C$ genes in the OsNAC7 subfamily under osmotic stress, salt stress, and in different tissues at different time points showed that members of the OsNAC7 subfamily not only played a regulatory role in the growth and development of roots, stems, and leaves of $C$. nankingense, but also responded to osmotic and salt stresses. These findings may provide new ideas for regulating plant stress resistance and growth. The function of $C H R 00069684$ was verified in transgenic tobacco plants, and it was found that $C H R 00069684$ could confer enhanced resistance to salt and low temperature stresses, improve the ABA sensitivity, and lead to the early flowering of transgenic tobacco. Studies on the CHR00069684 function confirmed the dual roles of the OsNAAC7 subfamily genes in stress regulation and plant growth and development. This study provides a theoretical basis for studying NAC TFs, the stress tolerance mechanism, and the plant growth process.

\section{References}

[1] Duan AQ, Yang XL,Feng K, Liu JX, Xu ZS, Xiong AS. 2019. Genome-wide analysis of NAC transcription factors and their response to abiotic stress in celery (Apium graveolens L.). Computational Biology and Chemistry 84: 107186 DOI: 10.1016/j.compbiolchem.2019.107186.

[2] Guerin C, Roche J, Allard V, Ravel C, Mouzeyar S, Bouzidi MF. 2019. Genome-wide analysis, expansion and expression of the NAC family under drought and heat stresses in bread wheat (T. aestivum L.). PLoS One 14(3): 19326203 DOI: 10.1371 journal.pone. 0213390

[3] Fang YJ, You J, Xie KB, Xie WB, Xiong L. 2008. Systematic sequence analysis and identification of tissuespecific or stress-responsive genes of NAC transcription factor family in rice. Molecular Genetics and Genomics 280(6): 547-563 DOI: 10.1007/s00438-008-0386-6

[4] Nuruzzaman M, Manimekalai R, Sharoni AM, Kouji Satoh K, Kondoh H, Ooka H, Kikuchi S. 2010. Genomewide analysis of NAC transcription factor family in rice. Gene 465: 30-40 DOI: 10.1016/j.gene.2010.06.008.

[5] Souer E, van Houwelingen A, Kloos D, Mol J, Koes R. 1996. The no apical meristem gene of Petunia is required for pattern formation in embryos and flowers and is expressed at meristem and primordia boundaries. Cell 85: 159170 DOI:10.1016/s0092-8674(00)81093-4.

[6] Aida M, Ishida T, Fukaki H, Fujisawa H, Tasaka M. 1997. Genes lnvolved in organ separation in Arabidopsis: an analysis of the cup-shaped cotyledon mutant. The Plant Cell 9: 841-857.

[7] Aida M, Ishida T, Tasaka M. 1999. Shoot apical meristem and cotyledon formation during Arabidopsis 
443

444

445

446

447

448

449

450

451

452

453

454

455

456

457

458

459

460

461

462

463

464

465

466

467

468

469

470

471

472

473

474

475

476

477

478

479

480

481

482

483

484

485

486

487

488

489

490

491

492

493

494

495

496

497

498

embryogenesis: interaction among the CUP-SHAPED COTYLEDON and SHOOT MERISTEMLESS genes. Development 126(8): 1563-1570.

[8] Ishida T, Aida M, Takada S, Tasaka M. 2000. Involvement of CUP-SHAPED COTYLEDON Genes in Gynoecium and Ovule Development in Arabidopsis thaliana. Plant and Cell Physiology 41(1): 60-67 DOI: 10.1093/pcp/41.1.60. [9] Ooka H, Satoh K, Doi K, Nagata T, Otomo Y, Murakami K, Matsubara K, Osato N, Kawai J, Carninci P, Hayashizaki Y, Suzuki K, Kojima K, Takahara Y, Yamamoto K, Kikuchi S. 2003. Comprehensive Analysis of NAC Family Genes in Oryza sativa and Arabidopsis thaliana. DNA Research 10 (6): 239-247 DOI: 10.1093/dnares/10.6.239.

[10] Liu H, Zhou Y, Li H, Wang T, Zhang J, Ouyang B, Ye Z. 2018. Molecular and functional characterization of ShNAC1, an NAC transcription factor from Solanum habrochaites. Plant Science 271: 9-19 DOI: 10.1016/j.plantsci.2018.03.005.

[11] Rushton PJ, Bokowiec MT, Han S, Zhang H, Brannock JF, Chen X, Laudeman TW, Timko MP. 2008. Tobacco transcription factors: novel insights into transcriptional regulation in the Solanaceae. Plant Physiology 147(1): 280295 DOI: 10.1104/pp.107.114041.

[12] Le DT, Nishiyama RIE, Watanabe Y, Mochida K, Yamaguchi-Shinozaki K, Shinozaki K, Tran L-SP. 2011. Genome-wide survey and expression analysis of the plant-specific NAC transcription factor family in soybean during development and dehydration stress. DNA Research 18(4): 263-276 DOI: 10.1093/dnares/dsr015.

[13] Su H, Zhang S, Yin Y, Zhu D, Han L. 2015. Genome-wide analysis of NAM-ATAF1,2-CUC2 transcription factor family in Solanum lycopersicum. Journal of Plant Biochemistry and Biotechnology 24(2): 176-183 DOI:10.1007/s13562-014-0255-9.

[14] Hu R, Qi G, Kong Y, Zhou G. 2010. Comprehensive Analysis of NAC Domain Transcription Factor Gene Family in Populus trichocarpa. BMC Plant Biology 145(10): 1471-2229 doi:10.1186/1471-2229-10-145.

[15] Ju YL, Yue XF, Min Z, Wang XH, Fang YL, Zhang JX. 2020. VvNAC17, a novel stress-responsive grapevine (Vitis vinifera L.) NAC transcription factor, increases sensitivity to abscisic acid and enhances salinity, freezing, and drought tolerance in transgenic Arabidopsis. Plant Physiology and Biochemistry 146: 98-111 DOI: 10.1016/j.plaphy.2019.11.002.

[16] Saidi MN, Mergby D, Brini F. 2017. Identification and expression analysis of the NAC transcription factor family in durum wheat (Triticum turgidum L. ssp. durum). Plant Physiology and Biochemistry 112: 117-128 DOI:10.1016/j.plaphy.2016.12.028.

[17] Ying Gao Y, Wei W, Zhao X, Tan X, Fan Z, Zhang Y, Jing Y, Meng L, Zhu B, Zhu H, Chen J, Jiang CZ, Grierson D, Luo Y, Fu DQ. 2018. A NAC transcription factor, NOR-like1, is a new positive regulator of tomato fruit ripening. Horticulture Research 75(5): 1-18 DOI: 10.1038/s41438-018-0111-5.

[18] Sun D, Zhang X, Zhang Q, Ji X, Jia Y, Wang H, Niu L, Zhang Y. 2019. Comparative transcriptome profiling uncovers a Lilium regale NAC transcription factor, LrNAC35, contributing to defence response against cucumber mosaic virus and tobacco mosaic virus. Molecular Plant Pathology 20(12): 1662-1681 DOI: 10.1111/mpp.12868.

[19] Puranik S, Sahu PP, Srivastava PS, Prasad M. 2012. NAC proteins: regulation and role in stress tolerance Trends in plant science 17(6): 369-381 DOI: 10.1016/j.tplants.2012.02.004.

[20] Xia N, Zhang G, Sun YF, Zhu L, Xu LS, Chen XM, Liu B, Yu YT, Wang XJ, Huang LL, Kang ZS. (2010) TaNAC8, a novel NAC transcription factor gene in wheat, responds to stripe rust pathogen infection and abiotic stresses. Physiological and Molecular Plant Pathology, 74, 394-402 DOI:10.1016/j.pmpp.2010.06.005

[21] Tran LSP, Nakashima K, Sakuma Y, Simpsonn SD, Fujita Y, Maruyama K, Fujita M, Seki M, Shinozaki K, Yamaguchi-Shinozak K. 2004. Isolation and functional analysis of Arabidopsis stress-inducible NAC transcription factors that bind to a drought-responsive cis-element in the early responsive to dehydration stress 1 promoter. The Plant cell 16: 2481-2498 DOI: 10.1105/tpc.104.022699.

[22] Wu Y, Deng Z, Lai J, Zhang Y, Yang C, Yin B, Zhao Q, Zhang L, Li Y, Yang C, Xie Q. 2009. Dual function of Arabidopsis ATAF1 in abiotic and biotic stress responses. Cell Research 19(11): 1279-1290 DOI: 10.1038/cr.2009.108.

[23] Jeong JS, Kim YS, Baek KH, Jung H, Ha SH, Choi YD, Kim M, Reuzeau C, Kim JK. 2010. Root-specific expression of OsNAC10 improves drought tolerance and grain yield in rice under field drought conditions. Plant physiology 153(1): 185-197 DOI: 10.1104/pp.110.154773.

[24] Nguyen KH, Mostofa MG, Li W, Van Ha C. 2018. The soybean transcription factor GmNAC085 enhances drought tolerance in Arabidopsis. Environmental and Experimental Botany 151: 12-20 DOI: 10.1016/j.envexpbot.2018.03.017.

[25] Yang X, Kim MY, Ha J, Lee SH. 2019. Overexpression of the Soybean NAC gene GmNAC109 increases lateral root formation and abiotic stress tolerance in transgenic Arabidopsis plants. Frontiers in Plant Science 10: 1036 DOI: 10.3389/fpls.2019.01036.

PeerJ reviewing PDF | (2021:01:57609:2:0:NEW 20 Apr 2021) 
499

500

501

502

503

504

505

506

507

508

509

510

511

512

513

514

515

516

517

518

519

520

521

522

523

524

525

526

527

528

529

530

531

532

533

534

535

536

537

538

539

540

541

542

543

544

545

546

547

548

549

550

551

552

553

554

[26] Hao YJ, Wei W, Song QX, Chen HW, Zhang YQ, Wang F, Zou HF, Lei G, Tian AG, Zhang WK, Ma B, Zhang JS, Chen SY. 2011. Soybean NAC transcription factors promote abiotic stress tolerance and lateral root formation in transgenic plants. Plant Journal 68(2): 302-313 DOI: 10.1111/j.1365-313X.2011.04687.x.

[27] Chen D, Chai S, McIntyre CL, Xue GP. 2018. Overexpression of a predominantly root-expressed NAC transcription factor in wheat roots enhances root length, biomass and drought tolerance. Plant Cell Reports 37(2): 225-237 DOI: 10.1007/s00299-017-2224-y.

[28] Huang Y, Li T, Xu ZS, Wang F, Xiong AS. 2017. Six NAC transcription factors involved in response to TYLCV infection in resistant and susceptible tomato cultivars. Plant Physiology and Biochemistry 120: 61-74 DOI: 10.1016/j.plaphy.2017.09.020.

[29] Selth LA, Dogra SC, Rasheed MS, Healy H, Randles JW, Rezaian MA. 2005. A NAC Domain Protein Interacts with Tomato leaf curl virus Replication Accessory Protein and Enhances Viral Replication. The Plant Cell 17(1): 311325 DOI: $10.1105 /$ tpc. 104.027235.

[30] Wang X, Goregaoker SP, Culver JN. 2009. Interaction of the Tobacco mosaic virus replicase protein with a NAC domain transcription factor is associated with the suppression of systemic host defenses. Journal of Virology 83(19): 9720-9730 DOI:10.1128/jvi.00941-09.

[31] Donze T, Qu F, Twigg P, Morris TJ. 2014. Turnip crinkle virus coat protein inhibits the basal immune response to virus invasion in Arabidopsis by binding to the NAC transcription factor TIP. Virology 449: 207-214 DOI: 10.1016/j.virol.2013.11.018.

[32] Liu H, Ouyang B, Zhang J, Wang T, Li H, Zhang Y, Yu C, Ye Z. 2012. Differential modulation of photosynthesis, signaling, and transcriptional regulation between tolerant and sensitive tomato genotypes under cold stress. PLoS One 7(11): e0050785 DOI: 10.1371/journal.pone.0050785.

[33] Nakashima K, Tran LSP, Van Nguyen D, Fujita M, Maruyama K, Todaka D, Ito Y, Hayashi N, Shinozaki K, Yamaguchi-Shinozaki K. 2007. Functional analysis of a NAC-type transcription factor OsNAC6 involved in abiotic and biotic stress-responsive gene expression in rice. The Plant Journal 51(4): 617-630 DOI: 10.1111/j.1365313X.2007.03168.X.

[34] Song SY, Chen Y, Chen J, Dai XY, Zhang WH. 2011. Physiological mechanisms underlying OsNAC5-dependent tolerance of rice plants to abiotic stress. Planta 234(2): 331-345 DOI: 10.1007/s00425-011-1403-2.

[35] Li L, Zheng T, Zhuo X, Li S, Qiu L, Wang J, Cheng T, Zhang Q. 2019. Genome-wide identification, characterization and expression analysis of the HD-Zip gene family in the stem development of the woody plant Prunus mume. PeerJ 7: e7499 DOI: 10.7717/peerj.7499.

[36] Hu W, Wei Y, Xia Z, Yan Y, Hou X, Zou M, Lu C, Wang W, Peng M. 2015. Genome-Wide Identification and Expression Analysis of the NAC Transcription Factor Family in Cassava. PLoS One 10(8): e0136993 DOI: 10.1371/journal.pone.0136993.

[37] Zhang J, Yang E, He Q, Lin M, Zhou W, Pian R, Chen X. 2019. Genome-wide analysis of the WRKY gene family in drumstick (Moringa oleifera Lam.). PeerJ 7: e7063 DOI:10.7717/peerj.7063.

[38] Li J, Gao K, Khan WU, Yang X, Yang X, Zhao T, Chen Z, An X. 2019. Genome-wide analysis of the poplar NF-Y gene family and its expression in floral bud development of Populus tomentosa. Trees 34(1): 285-296 DOI: 10.1007/s00468-019-01917-3.

[39] Kelley LA, Mezulis S, Yates CM, Wass MN and Sternberg MJ. 2015. The Phyre2 web portal for protein modeling, prediction and analysis. Nature Protocols 10(6): 845-858 DOI: 10.1038/nprot.2015.053.

[40] Fan Q, Song A, Jiang J, Zhang T, Sun H, Wang Y, Chen S, Chen F. 2016. CmWRKY1 Enhances the Dehydration Tolerance of Chrysanthemum through the Regulation of ABA-Associated Genes. PLoS One 11(3): e0150572 DOI: 10.1371/journal.pone.0150572.

[41] Chen J, Shen CZ, Guo YP, Rao GY. 2018. Patterning the Asteraceae Capitulum: Duplications and Differential Expression of the Flower Symmetry CYC2-Like Genes. Frontiers in Plant Science 9:551 DOI: 10.3389/fpls.2018.00551.

[42] Ewei Y, Esh H, Exia Z, Etie W, Eding Z, Eyan Y, Ewang W, Ehu W, Eli K. 2016. Genome-wide identification and expression analysis of the WRKY gene family in Cassava. Frontiers in Plant Science. 7: 25 DOI: $10.3389 /$ fpls.2016.00025.

[43] R. B. Horsch, J. E. Fry, N. L. Hoffmann, D. Eichholtz, S. G. Rogers and R. T. Fraley (1985) A Simple and General Method for Transferring Genes into Plants. Science, New Series 227(4691): 1229-1231 DOI: $10.2307 / 1694217$

[44] Chen QF, Wang Q, Xiong LZ, Lou ZY. 2011. A structural view of the conserved domain of rice stress-responsive NAC1. Protein Cell 2(1): 55-63 DOI: 10.1007/s13238-011-1010-9

[45] Heidi A.E, Addie NO, Karen S, Sine L, Leila LL. 2004. Structure of the conserved domain of ANAC, a member of the NAC family of transcription factors. EMBO REPORTS 5(3): 297-303 DOI:10.1038/sj.embor.7400093

Peer) reviewing PDF | (2021:01:57609:2:0:NEW 20 Apr 2021) 
555

556

557

558

559

560

561

562

563

564

565

566

567

568

569

570

571

572

573

574

575

576

577

578

579

580

581

582

583

584

585

586

587

588

589

590

591

592

593

594

595

596

597

598

599

600

601

602

603

604

605

606

607

608

609

610

[46] Zhu YX, Yang L, Liu N, Yang J, Zhou XK, Xia YC, He Y, He YQ, Gong HJ, Ma DF, Yin JL. 2019. Genomewide identification, structure characterization, and expression pattern profiling of aquaporin gene family in cucumber. BMC Plant Biology 19(1): 345 DOI: 10.1186/s12870-019-1953-1.

[47] Masatoshi Y, Minoru K, Hiroo F, Taku D. 2008. VASCULAR-RELATED NAC-DOMAIN7 is involved in the differentiation of all types of xylem vessels in Arabidopsis roots and shoots. The Plant Journal 55, 652-664 DOI: 10.1111/j.1365-313X.2008.03533.x

[48] Sakuraba Y, Piao W, Lim JH, Han SH, Kim YS, An G, Paek NC. 2015. Rice ONAC106 inhibits leaf senescence and increases salt tolerance and tiller angle. Plant Cell Physiology. 56(12): 2325-2339 DOI: 10.1093/pcp/pcv144.

[49] Sun L, Huang L, Hong Y, Zhang H, Song F, Li D. 2015. Comprehensive analysis suggests overlapping expression of rice ONAC transcription factors in abiotic and biotic stress responses. International Journal of Molecular Sciences 16(2): 4306-4326 DOI:10.3390/ijms16024306.

[50] Zhong R, Demura T, Ye ZH. 2006. SND1, a NAC domain transcription factor, is a key regulator of secondary wall synthesis in fibers of Arabidopsis. The Plant Cell 18(11): 3158-3170. DOI:10.1105/tpc.106.047399.

[51] Mitsuda N, Iwase A, Yamamoto H, Yoshid M, Seki M, Shinozaki K, Ohme-Takagi M. 2007. NAC transcription factors, NST1 and NST3, are key regulators of the formation of secondary walls in woody tissues of Arabidopsis. Plant Cell. 19(1): 270-280 DOI: 10.1105/tpc.106.047043.

[52] Zhong R, Ye ZH. 2015. The Arabidopsis NAC transcription factor NST2 functions together with SND1 and NST1 to regulate secondary wall biosynthesis in fibers of inflorescence stems. Plant Signaling \& Behavior 10(2): e989746 DOI: 10.4161/15592324.2014.989746.

[53] Fendrych M, Van Hautegem T, Van Durme M, Olvera-Carrillo Y, Huysmans M, Karimi M, Lippens S, Guerin CJ, Krebs M, Schumacher K, Nowack MK. 2014. Programmed cell death controlled by ANAC033/SOMBRERO determines root cap organ size in Arabidopsis. Current Biology 24(9): 931-40 DOI: 10.1016/j.cub.2014.03.025.

[54] Bennett T, Van Den Toorn A, Sanchez-Perez GF, Campilho A, Willemsen V, Snel B, Scheres B. 2010. SOMBRERO, BEARSKIN1, and BEARSKIN2 regulate root cap maturation in Arabidopsis. Plant Cell 22(3): 64054 DOI: $10.1105 /$ tpc. 109.072272 .

[55] Zhou J, Zhong R, Ye ZH. 2014. Arabidopsis NAC Domain Proteins, VND1 to VND5, Are Transcriptional Regulators of Secondary Wall Biosynthesis in Vessels. PLoS One. 9(8): e105726 DOI: 10.1371/journal.pone.0105726.

[56] Endo H, Yamaguchi M, Tamura T. 2015. Multiple Classes of Transcription Factors Regulate the Expression of VASCULARRELATED NAC-DOMAIN7, a Master switch of xylem vessel differentiation. Plant Cell Physiology 56 (2): 242-254 DOI: $10.1093 / \mathrm{pcp} / \mathrm{pcu} 134$.

[57] Soyano T, Thitamadee S, Machida Y, Chua NH. 2008. ASYMMETRIC LEAVES2- LIKE19/LATERAL ORGAN BOUNDARIES DOMAIN30 and ASL20/LBD18 regulate tracheary element differentiation in Arabidopsis. Plant Cell. 20: 3359-3373 DOI: $10.1105 /$ tpc. 108.061796.

[58] Yamaguchi M, Ohtani M, Mitsuda N, Kubo M. 2010. VNDINTERACTING2, a NAC domain transcription factor, negatively regulates xylem vessel formation in Arabidopsis. Plant Cell 22: 1249-1263 DOI: 10.1105/tpc.108.064048. [598] Yang Y, Zhu K, Wu J, Liu L, Sun G, He Y, Chen F, Yu D. 2016. Identification and characterization of a novel NAC-like gene in chrysanthemum (Dendranthema nankingense). Plant Cell Reports 35(8): 1783-1798 DOI: 10.1007/s00299-016-1996-9.

[60] Liu QL, Xu KD, Zhao LJ, Pan YZ, Jiang BB, Zhang HQ, Liu GL. 2011. Overexpression of a novel chrysanthemum NAC transcription factor gene enhances salt tolerance in tobacco. Biotechnology Letters 33(10): 2073-2082 DOI: 10.1007/s10529-011-0659-8.

[61] Jeong JS, Kim YS, Redillas MC, Jang G, Jung H, Bang SW, Choi YD, Ha SH, Reuzeau C, Kim JK. 2013. OsNAC5 overexpression enlarges root diameter in rice plants leading to enhanced drought tolerance and increased grain yield in the field. Plant Biotechnology Journal 11(1): 101-114 DOI: 10.1111/pbi.12011.

[62] Rachmat A, Nugroho S, Sukma D, Aswidinnoor H, Sudarsono S. 2014. Overexpression of OsNAC6 transcription factor from Indonesia rice cultivar enhances drought and salt tolerance. Emirates Journal of Food and Agriculture. 26(6):519-527 DOI: 10.9755/ejfa.v26i6.17672.

[63] Chung PJ, Kim YS, Jeong JS, Park SH, Nahm BH, Kim JK. 2009. The histone deacetylase OsHDAC1 epigenetically regulates the OsNAC6 gene that controls seedling root growth in rice. Plant Journal 59(5): 764-776 DOI: $10.1111 / \mathrm{j} .1365-313 X .2009 .03908 . x$.

[64] Yokotani N, Ichikawa T, Kondou Y, Matsui M, Hirochika H, Iwabuchi M, Oda K. 2009. Tolerance to various environmental stresses conferred by the salt-responsive rice gene ONAC063 in transgenic Arabidopsis. Planta 229(5): 1065-1075 DOI: 10.1007/s00425-009-0895-5.

[65] Hong Y, Zhang H, Huang L, Li D, Song F. 2016. Overexpression of a Stress-Responsive NAC Transcription Factor Gene ONAC022 Improves Drought and Salt Tolerance in Rice. Frontiers in Plant Science 7: 4 DOI:

Peer) reviewing PDF | (2021:01:57609:2:0:NEW 20 Apr 2021) 
611

612

613

614

615

616

617

618

619

620

621

622

623

624

625

626

627

628

629

630

631

632

633

634

635

636

637

638

639

640

641

642

643

644

645

646

647

648

649

650

651

652

653

654

655

656

10.3389/fpls.2016.00004.

[66] Wang Z, Xia Y, Lin S, Wang Y, Guo B, Song X, Ding S, Zheng L, Feng R, Chen S, Bao Y, Sheng C, Zhang X, Wu J, Niu D, Jin H, Zhao H. 2018. Osa-miR164a targets OsNAC60 and negatively regulates rice immunity against the blast fungus Magnaporthe oryzae. The Plant Journal 95:584-597 DOI: 10.1111/tpj.13972.

[67] Mao C, Lu S, Lv B, Zhang B, Shen J, He J, Luo L, Xi D, Chen X, Ming F. 2017. A Rice NAC Transcription Factor Promotes Leaf Senescence via ABA Biosynthesis. Plant Physiology 174(3): 1747-1763 DOI: 10.1104/pp.17.00542.

[68] Shen J, Lv B, Luo L, He J, Mao C, Xi D, Ming F. 2017. The NAC-type transcription factor OsNAC2 regulates ABA-dependent genes and abiotic stress tolerance in rice. Scientific Reports. 7(1):40641 DOI:10.1038/srep40641.

[69] Chen X, Lu S, Wang Y, Zhang X, Lv B, Luo L, Xi D, Shen J, Ma H, Ming F. 2015. OsNAC2 encoding a NAC transcription factor that affects plant height through mediating the gibberellic acid pathway in rice. The Plant Journal. 82(2): 302-314 DOI: 10.1111/tpj.12819.

[70] Zheng X, Chen B, Lu G, Han B. 2009. Overexpression of a NAC transcription factor enhances rice drought and salt tolerance. Biochemical and Biophysical Research Communications 379(4): 985-989 DOI: 10.1016/j.bbrc.2008.12.163.

[71] Jeong JS, Kim YS, Baek KH, Jung H, Ha SH, Do Choi Y, Kim M, Reuzeau C, Kim JK. 2010. Root-specific expression of OsNAC10 improves drought tolerance and grain yield in rice under field drought conditions. Plant Physiology 153(1): 185-197 DOI: 10.1104/pp.110.154773.

[72] Chen X, Wang Y, Lv B, Li J, Luo L, Lu S, Zhang X, Ma H, Ming F. 2014. The NAC family transcription factor OsNAP confers abiotic stress response through the ABA pathway. Plant and Cell Physiology 55(3): 604-619 DOI: $10.1093 / \mathrm{pcp} / \mathrm{pct} 204$.

[73] Bo W, Zhaohui Z, Huanhuan Z, Xia W, Binglin L, Lijia Y, Xiangyan H, Deshui Y, Xuelian Z, Chunguo W, Wenqin S, Chengbin C, Yong Z. 2019. Targeted Mutagenesis of NAC Transcription Factor Gene, OsNAC041, Leading to Salt Sensitivity in Rice. Rice Science 26(2): 98-108 DOI: 10.1016/j.rsci.2018.12.005.

[74] Gao F, Xiong A, Peng R, Jin X, Xu J, Zhu B, Chen J, Yao Q. 2009. OsNAC52, a rice NAC transcription factor, potentially responds to ABA and confers drought tolerance in transgenic plants. Plant Cell, Tissue and Organ Culture (PCTOC) 100(3): 255-262 DOI: 10.1007/s11240-009-9640-9.

[75] Sun L, Zhang H, Li D, Huang L, Hong Y, Ding XS, Nelson RS, Zhou X, Song F. 2013. Functions of rice NAC transcriptional factors, ONAC122 and ONAC131, in defense responses against Magnaporthe grisea. Plant Molecular Biology 81(1-2): 41-56 DOI: 10.1007/s11103-012-9981-3.

[76] Dong F, Huang H, Liu J, Zhang M, ZhouY, Dai S. 2018. Cloning and function analysis of CnNAC9 from Chrysanthemum nankingense. Canadian Journal of Plant Science 98(6): 1265-1279 DOI: 10.1139/cjps-2017-0349.

[77] Liu Y, He M. 2019. The Chrysanthemum nankingense CnNAC9 gene positively regulates saline, alkaline, and drought stress in transgenic Chrysanthemum grandiflora. Journal of the American Society for Horticultural Science 144(4): 280-288 DOI: $10.21273 /$ jashs04697-19.

[78] Liu R, Wu M, Liu HL, Gao YM, Chen J, Yan HW, Xiang Y. 2020. Genome-wide identification and expression analysis of the NF-Y transcription factor family in Populus. Physiologia Plantarum 0:1-19 DOI: 10.1111/ppl.13084. [79] Kubo M, Udagawa M, Nishikubo N, Horiguchi G, Yamaguchi M, Ito J, Mimura T, Fukuda H, Demura T. 2005. Transcription switches for protoxylem and metaxylem vessel formation. Genes Dev. 19(16): 1855-1860 DOI: 10.1101/gad.1331305.

[80] Ko JH, Yang SH, Park AH, Lerouxel O, Han KH. 2007. ANAC012, a member of the plant-specific NAC transcription factor family, negatively regulates xylary fiber development in Arabidopsis thaliana. The Plant Jouranl. 50(6): 1035-1048 DOI: 10.1111/j.1365-313X.2007.03109.x.

[81] Kubo M, Udagawa M, Nishikubo N, Horiguchi G, Yamaguchi M, Ito J, Mimura T, Fukuda H, Demura T. 2005. Transcription switches for protoxylem and metaxylem vessel formation. Genes \& Development. 19(16): 1855-1860 DOI:10.1101/gad.1331305.

Peer] reviewing PDF | (2021:01:57609:2:0:NEW 20 Apr 2021) 


\section{Figure 1}

Phylogenetic tree analysis of NAC transcription factors from A. thaliana, 0 . sativa, and C. nankingense.

The phylogenetic trees were derived using the neighbor-joining (NJ) method by 1000 bootstrap replications. The black circles on the outer ring represent Group1 and Group2. Each color inside represents a subfamily. The red dots represent $C$. nankingense. The blue dots represent rice. The unmarked represents Arabidopsis thaliana. Numbers on the branches indicate differences between different genes, and higher numbers indicate higher relatedness. 


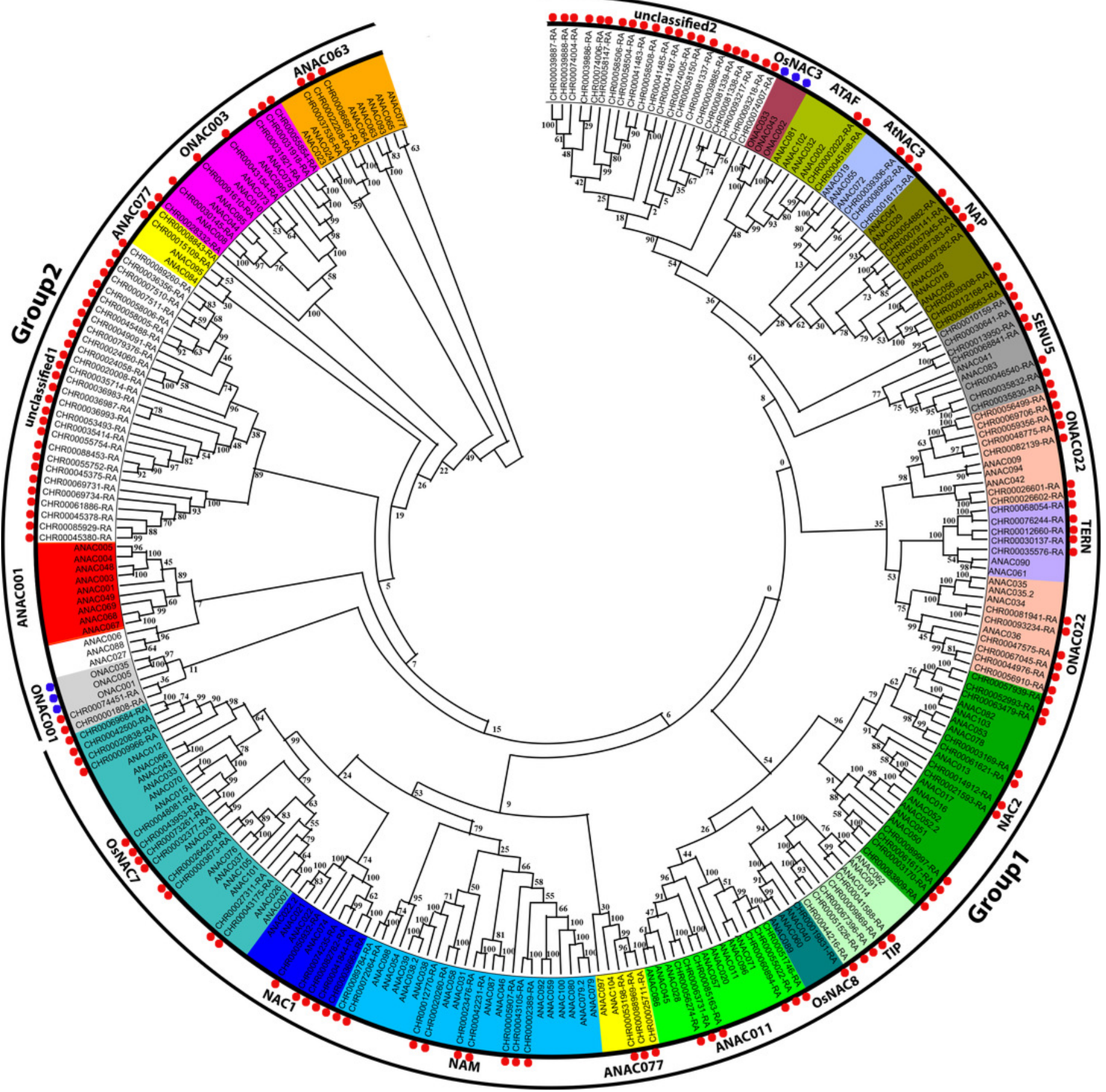




\section{Figure 2}

Predicted three-dimensional structure of NAC TFs from C. nankingense.

(A) Protein structure of different subfamilies in Group1. (B) Protein structure of different subfamilies in Group2. One gene from each branch of NAC phylogenetic trees is selected for prediction (Fig1). All genes of the OsNAC7 subfamily were predicted and compared with members of the OsNAC7 subfamily of $A$. thaliana NAC TFs.
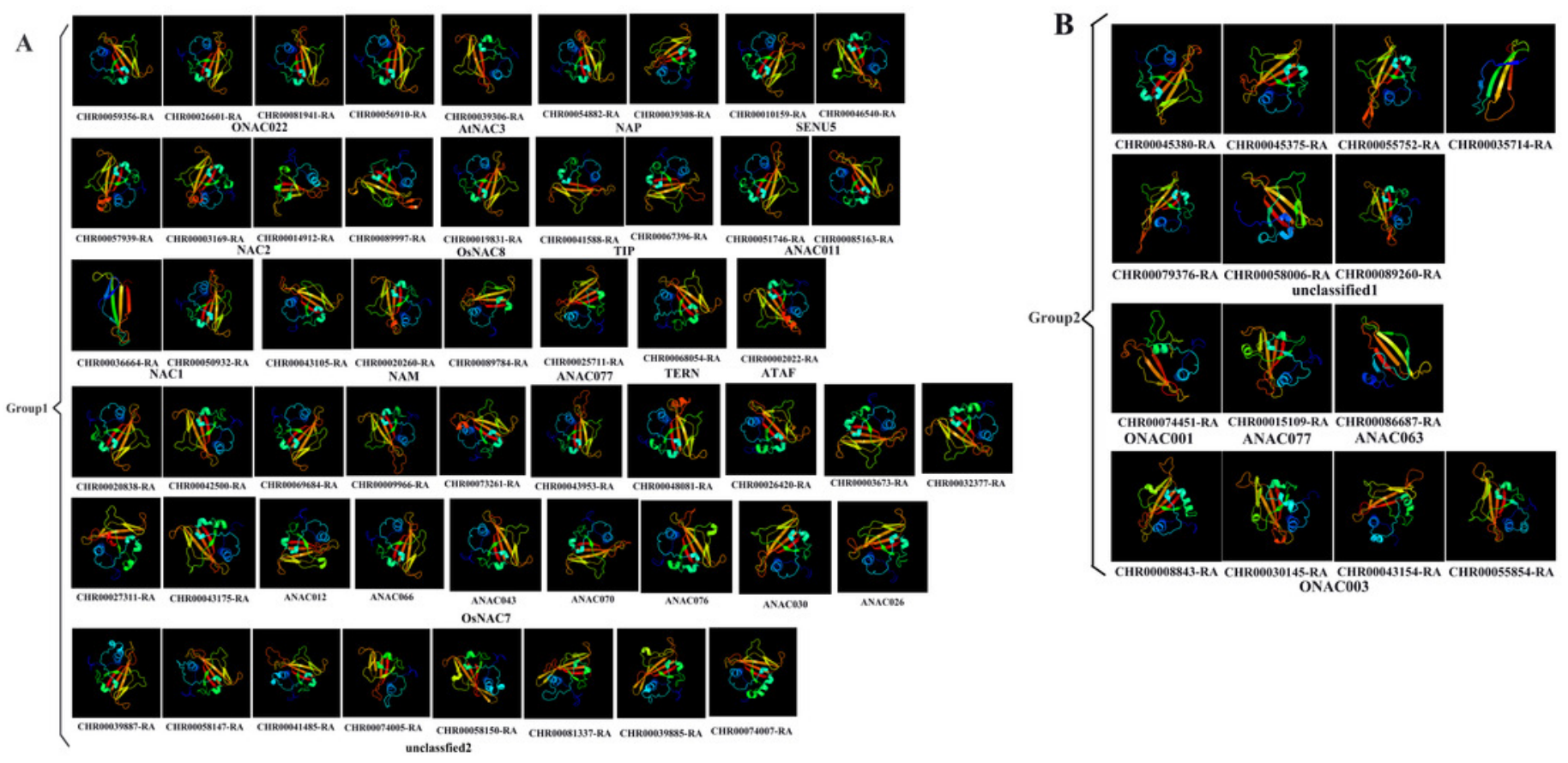


\section{Figure 3}

Sequence logos of conserved domains in C. nankingense.

The overall height of the stack represents the level of sequence conservation. Heights of residues within a stack indicate the frequency of each residue at that site. E-value (Expect value) is an estimate of the expected number of motifs. Sites are the number of sites contributing to the construction of the motif. Width shows the motif, each motif describes a pattern of a fixed width. 


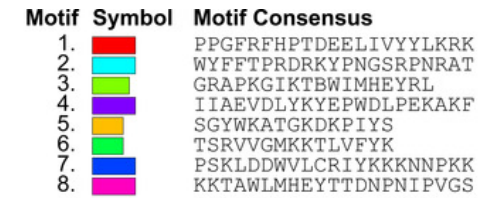

1. AfA DELW LW

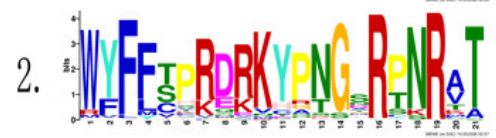

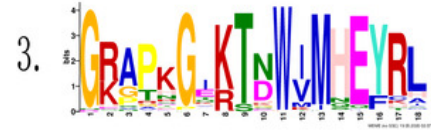

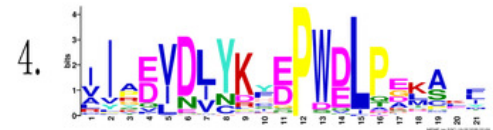

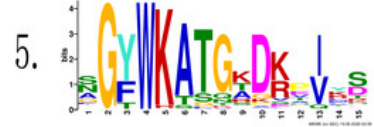

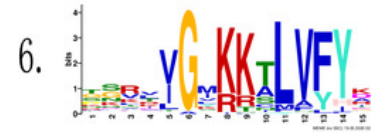

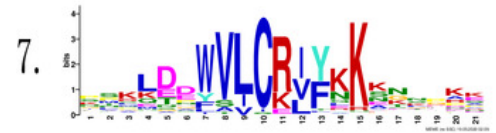

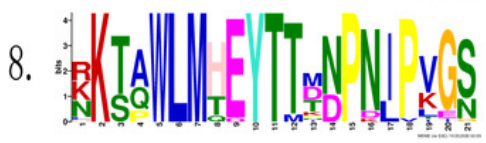

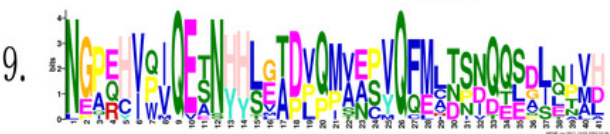

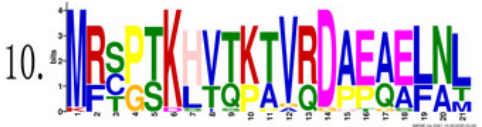

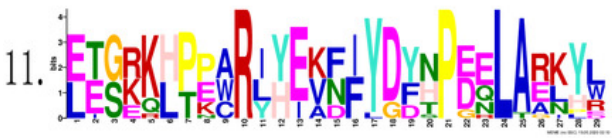

12.

13.

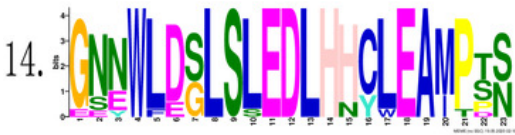

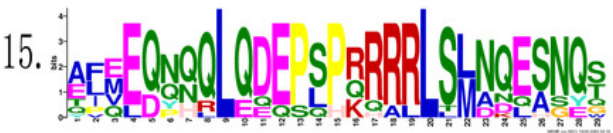

NGPEHVPIQESNHHLEADVQMVEPVQFMLTSBQQSDLNIVH

MRSPTKHVTKTVRDAEAELNL

YYRASPASTNGFRSSGASSSSKVS

YY

GNNWLDSISLEDLHHCLEAMPTS

AFEEQNQQLQDEPSPRRRRLSLNQESNQS

E-value

Sites

1. $1 e^{-1914} \quad 129$

119

21

1. $3 e^{-1721}$

$6.8 e^{-1361}$

110

18

5. 4e-1032

109

21

4. $1 e^{-1008}$

131

15

1. 1e-854

139

15

9. $0 e^{-811}$

119

21

3. $2 e^{-}-339$

25

21

1. 5e-299

16

41

3. 1e-247

22

21

5. 1e-208

16

29

2. 0e-198

13

29

2. 0e-191

12

38

1. $6 \mathrm{e}-159$

12

23

3. $3 e^{-182}$

15 
Figure 4

Conserved motifs analyses of NAC TFs in C. nankingense.

Different colors represent different motifs. P-value is the probability value test. Motif Locations shows the location of motif sites. 


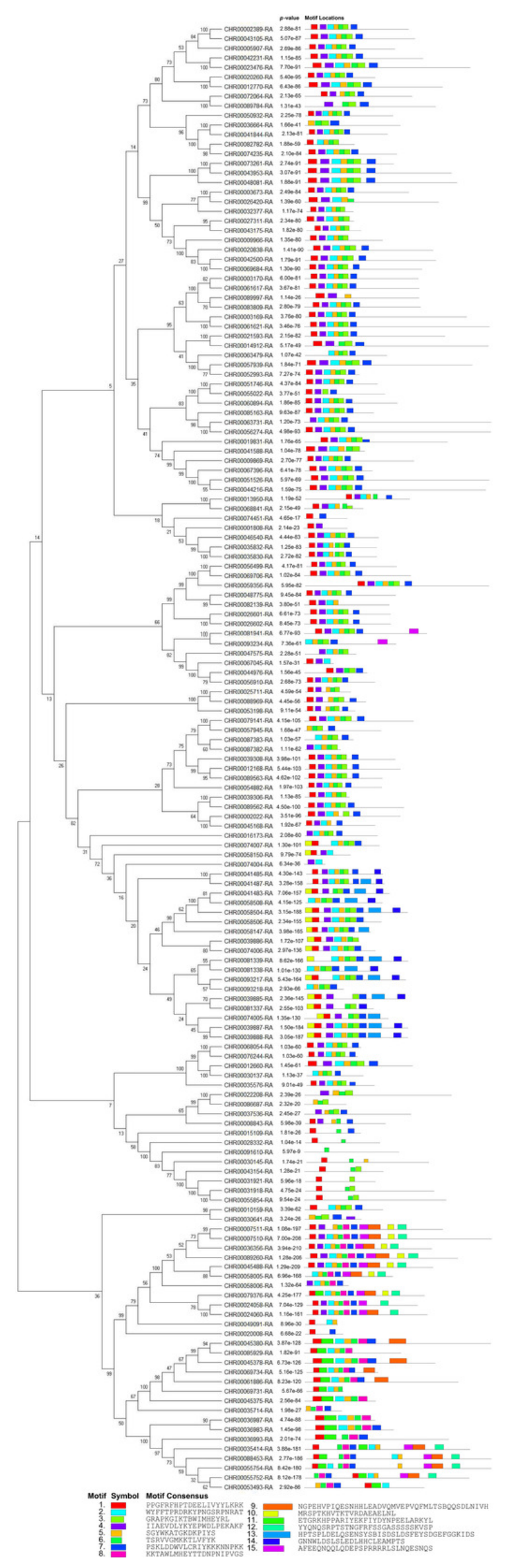

Peer) reviewing PDF | (2021:01:57609:2:0:NEW 20 Apr 2021) 


\section{Figure 5}

The morphology of $C$. nankingense at different growth stages.

(A) The seedling period 28 days after sowing. (B) The rapid growth period 44 days after sowing. (C) The stable growth and aging period 60 days after sowing.

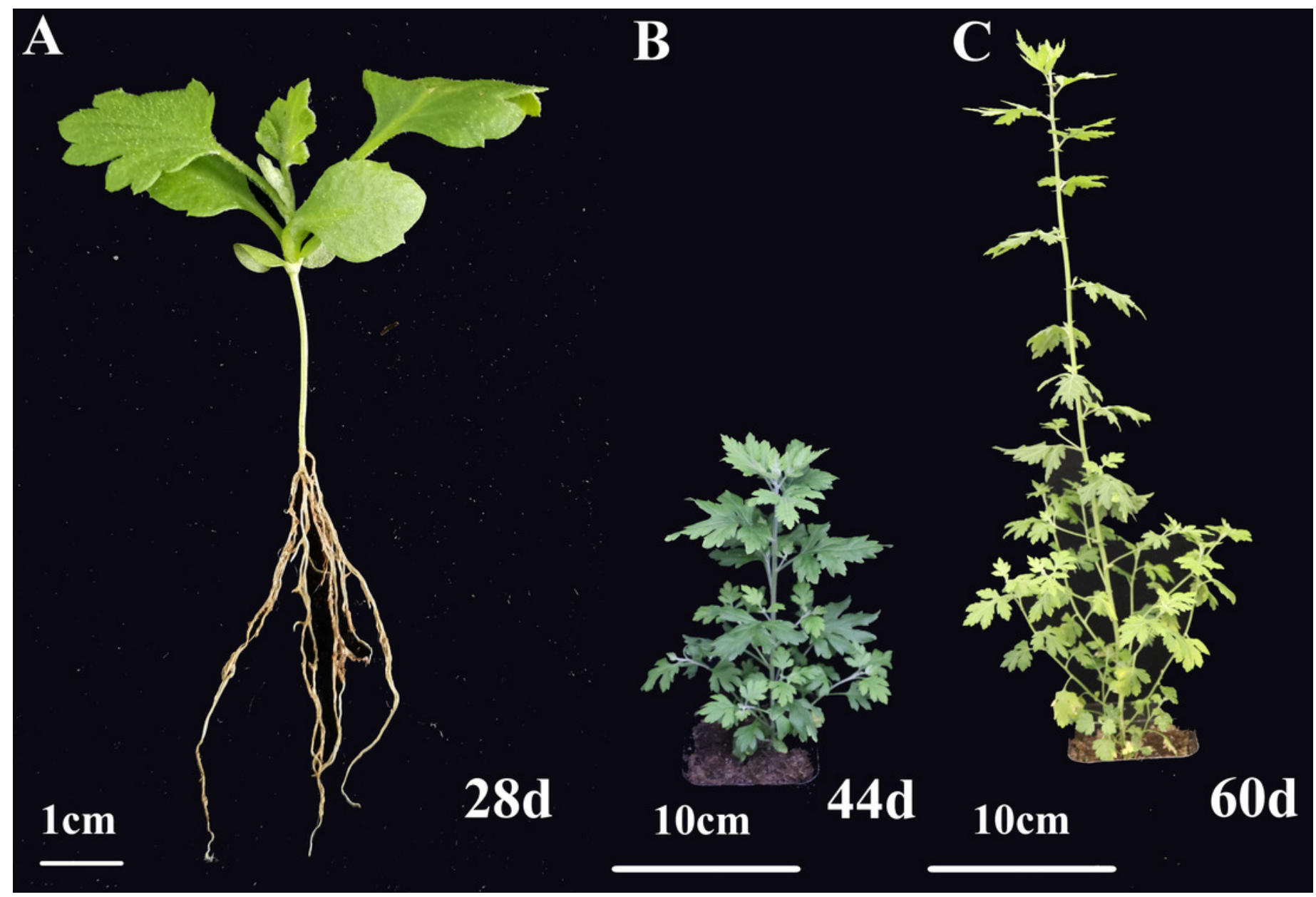




\section{Figure 6}

Expression pattern analysis of 12 OsNAC7 subfamily genes in different tissues of $C$. nankingense at different growth stages.

The expression of root at $28 \mathrm{~d}$ was used as a reference. The $\mathrm{x}$-coordinate represents the different tissues. The $y$-coordinate represents the expression level. Different color columns indicate different growth periods. Each diagram represents a different gene. Different lower case letters indicate statistically significant differences at the $\mathrm{P}<0.05$ level. 
A

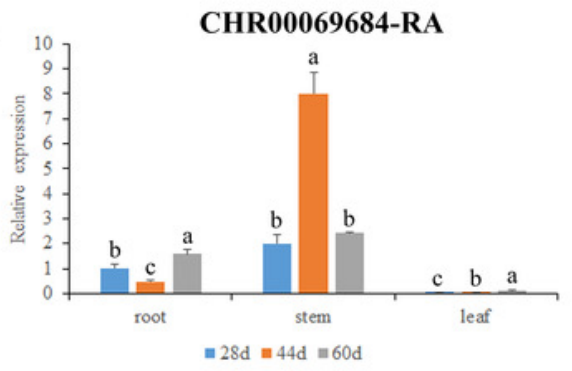

D

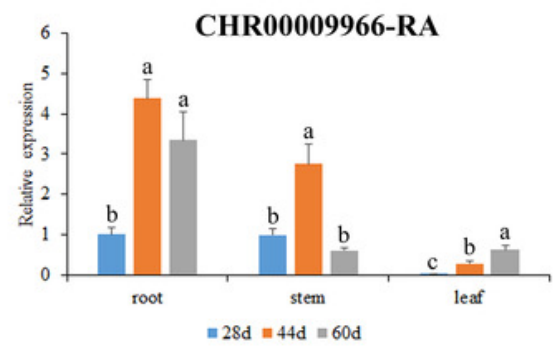

G

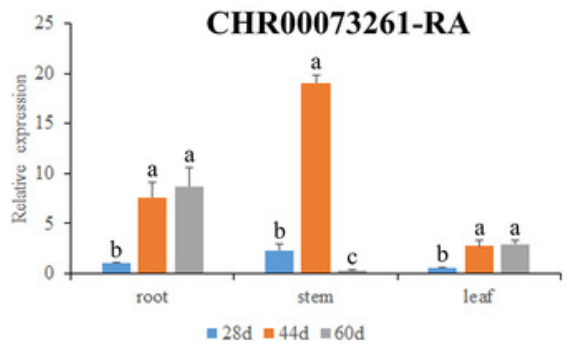

$\mathbf{J}$

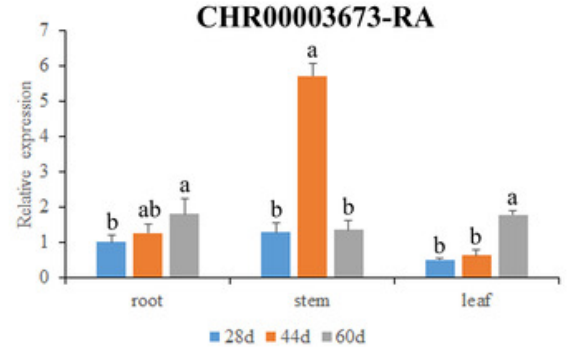

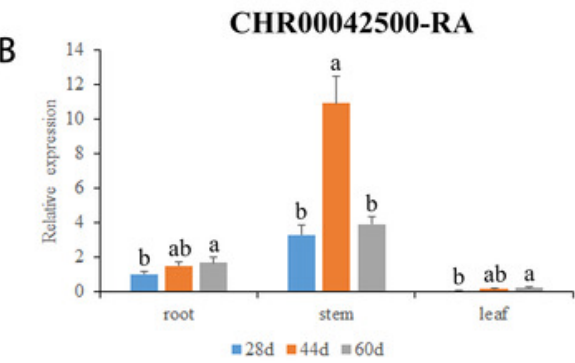

E

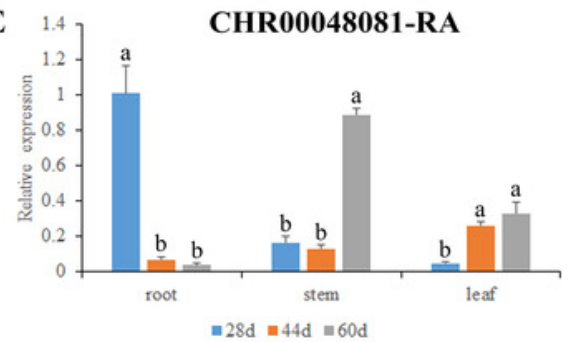

H

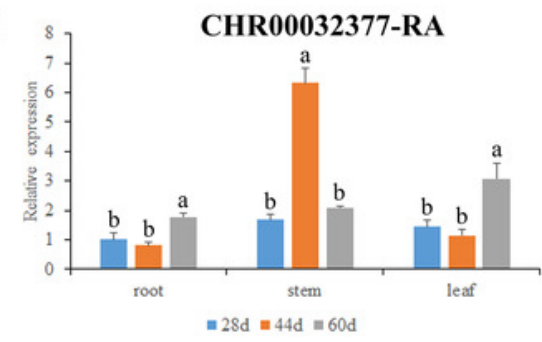

$\mathbf{K}$

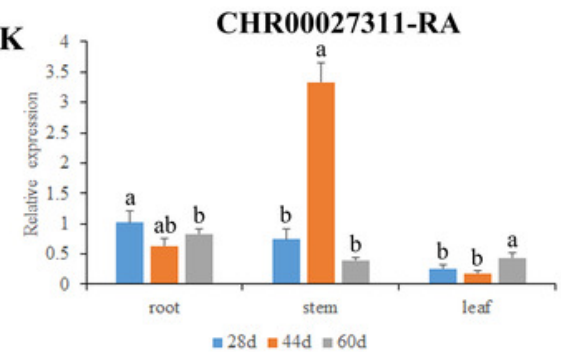

CHR00020838-RA

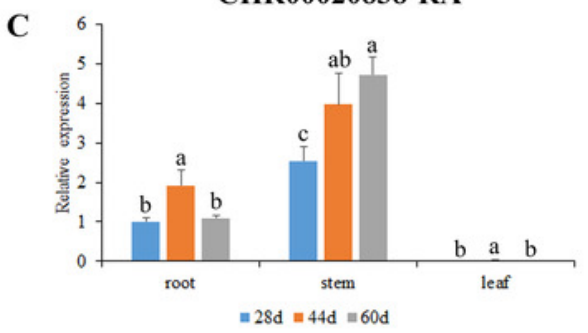

F

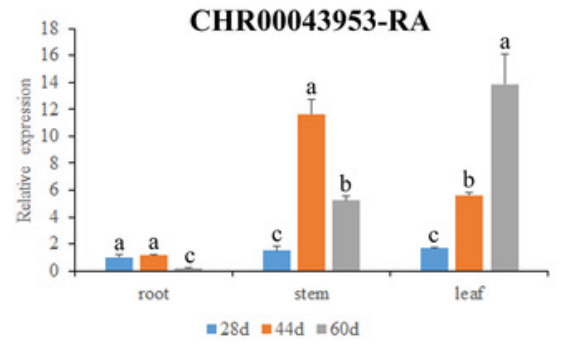

I $\quad 25] \quad$ CHR00026420-RA

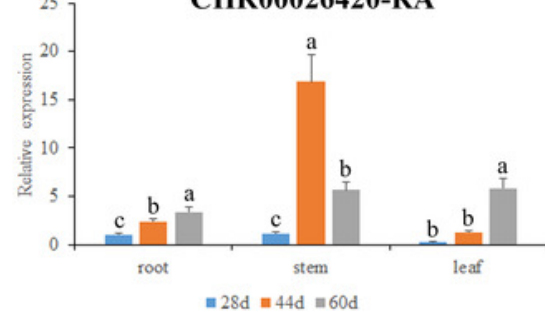

L

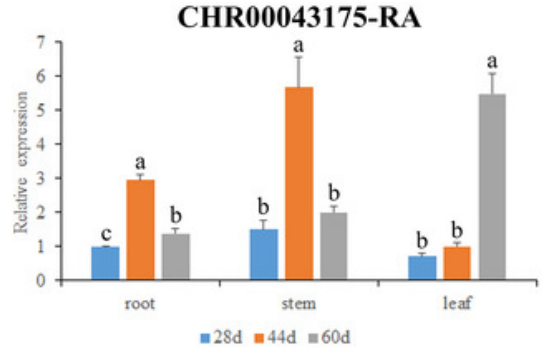




\section{Figure 7}

Expression pattern analysis of 12 OsNAC7 subfamily genes in $C$. nankingense under different treatment times of osmotic stress induced by $20 \%$ PEG6000.

The expression level of $0 \mathrm{~h}$ was used as a reference. The $\mathrm{x}$-coordinate represents the processing time. The $y$-coordinate represents the expression level. Each diagram represents a different gene. Different lower case letters indicate statistically significant differences at the $\mathrm{P}<0.05$ level. 
A

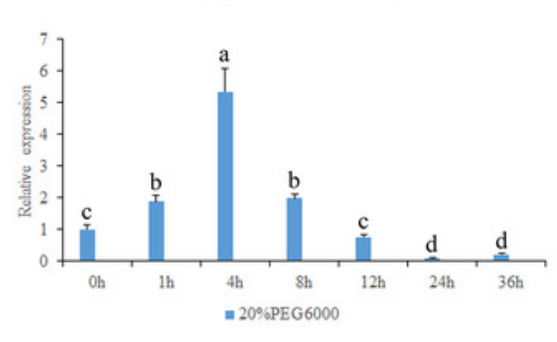

D

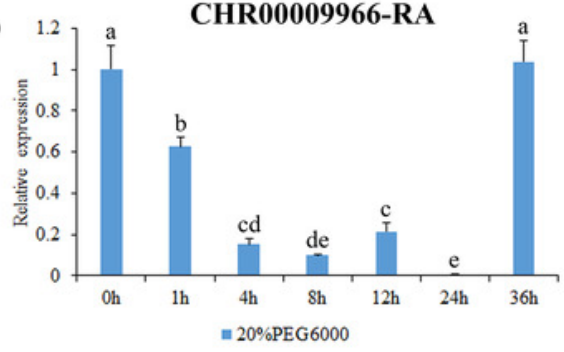

G
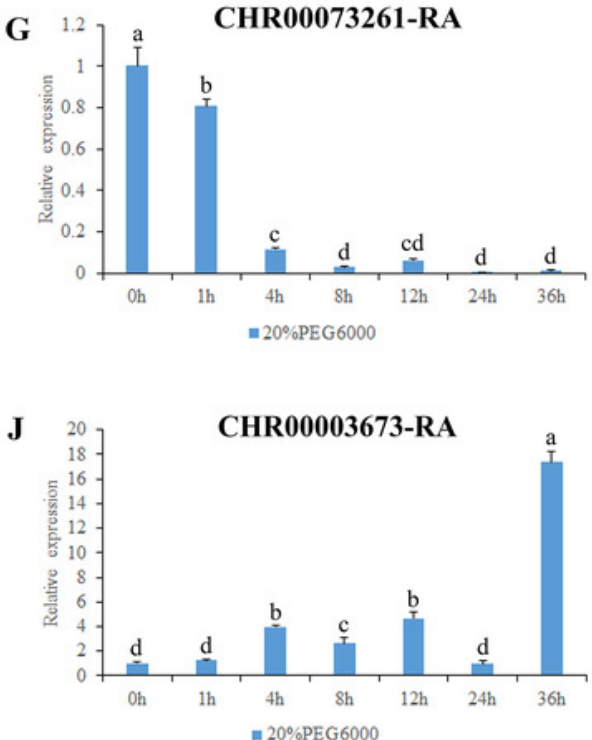

B

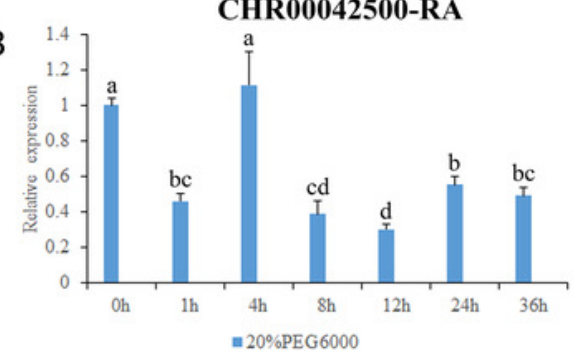

E
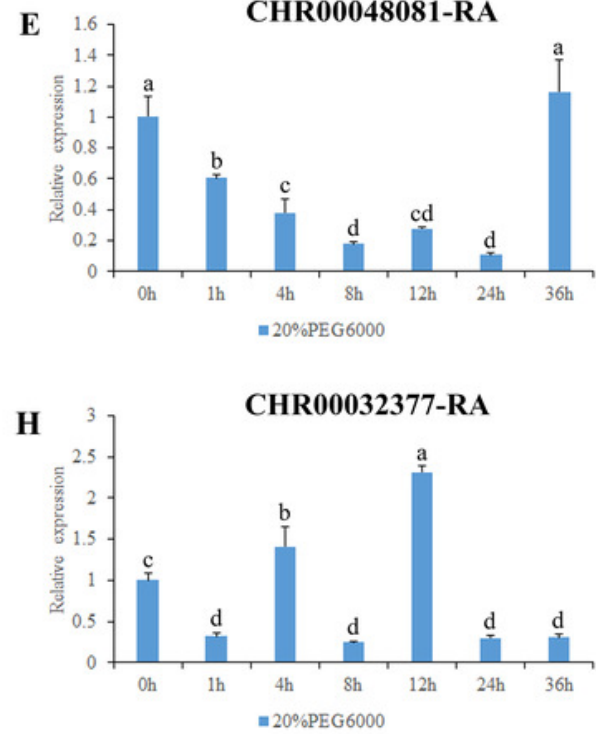

K

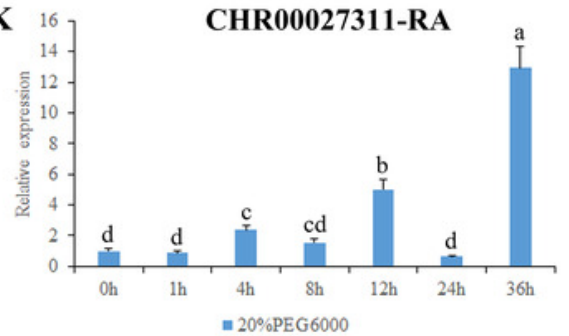

CHR00020838-RA
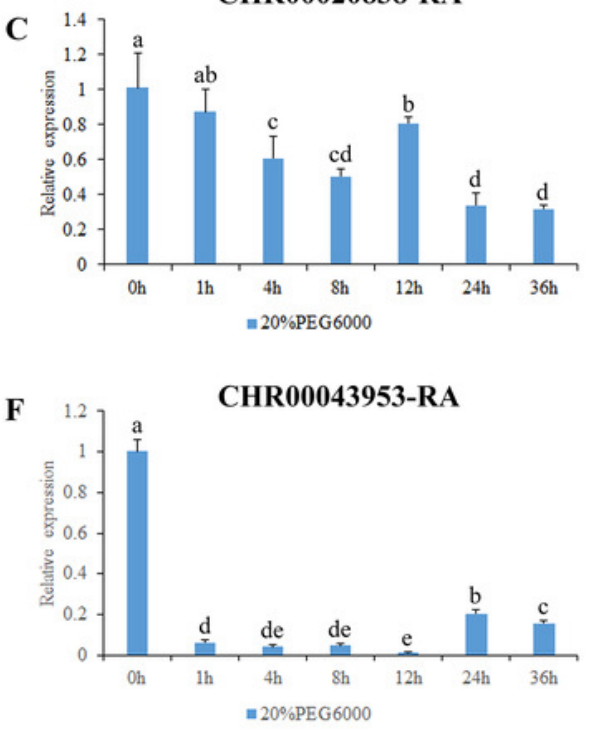

I

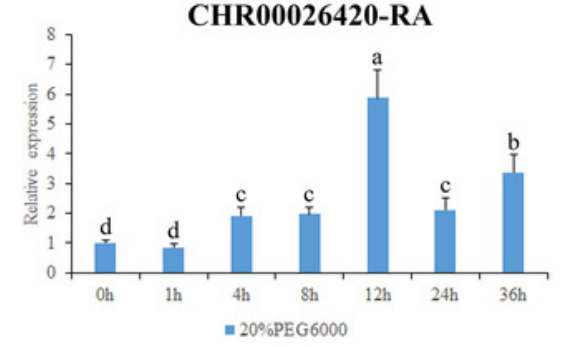

$\mathbf{L}$

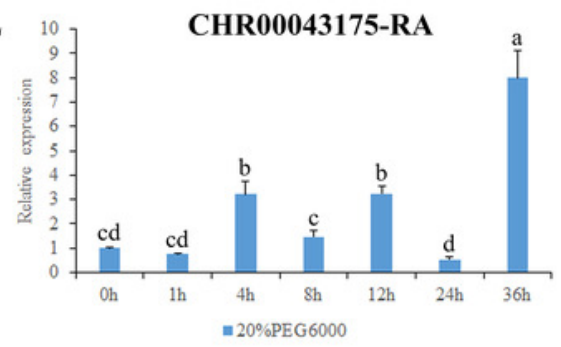


Figure 8

Expression pattern analysis of 12 OsNAC7 subfamily genes in C. nankingense under different treatment times of salt stress induced by $200 \mathrm{mM} \mathrm{NaCl}$.

The expression level of $0 \mathrm{~h}$ was used as a reference. The $\mathrm{x}$-coordinate represents the processing time. The $y$-coordinate represents the expression level. Each diagram represents a different gene. Different lower case letters indicate statistically significant differences at the $\mathrm{P}<0.05$ level.

A
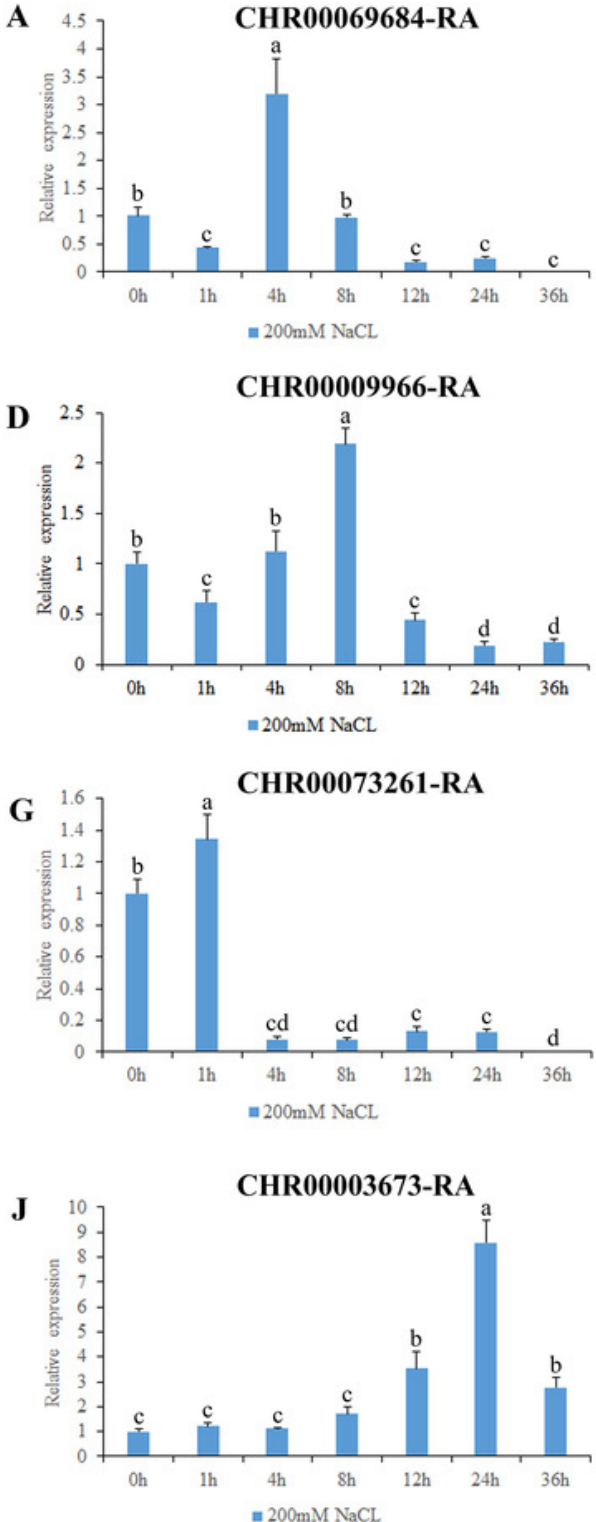

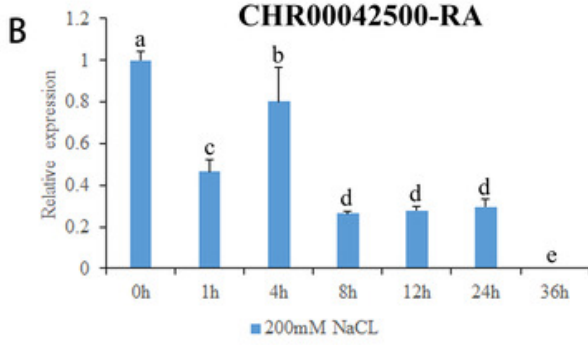

CHR00048081-RA

$\mathbf{E}$

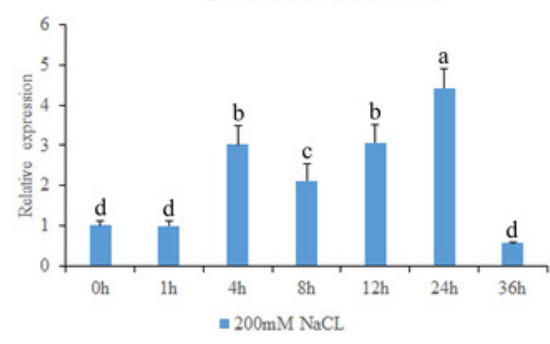

CHR00032377-RA

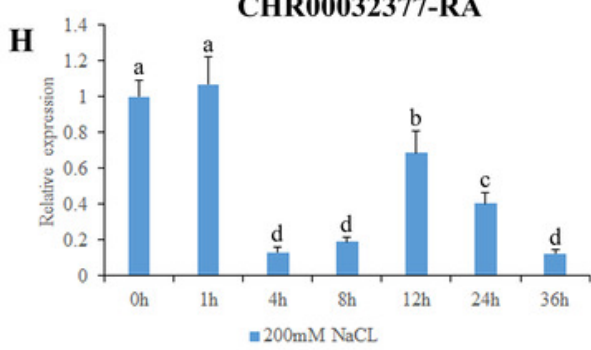

$\mathbf{K}$

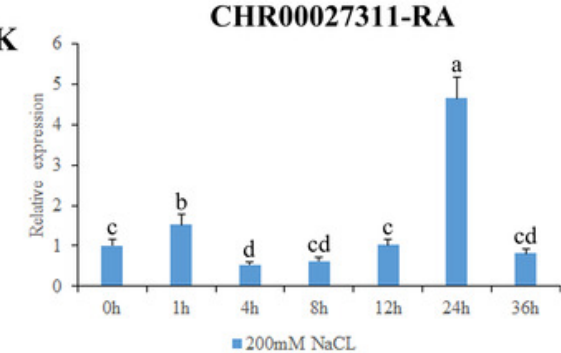

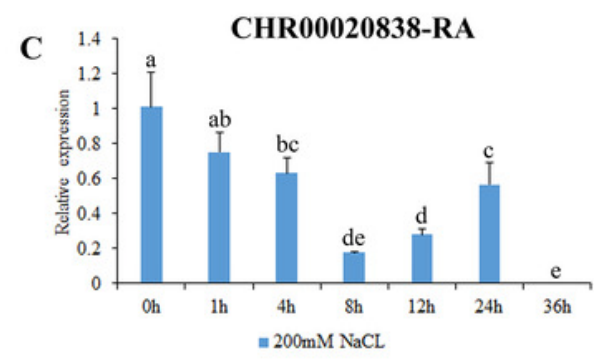

CHR00043953-RA

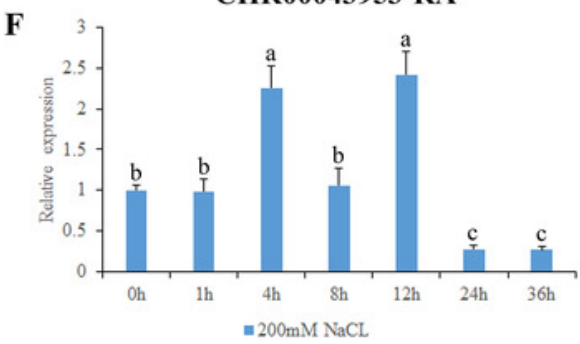

CHR00026420-RA

I

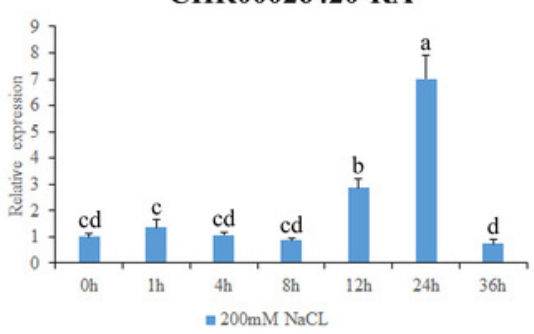

CHR00043175-RA

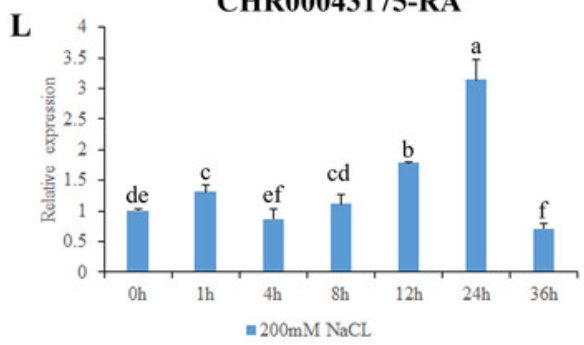




\section{Figure 9}

Subcellular localization of transgenic tobacco.

(A-D) Subcellular localization of super35S::GFP protein in root (A), leaf (B), root tip (C), and stem (D). (a-d) Enlarged image of GFP in root (a), leaf (b), root tip (c), and stem (d). (E, F) Subcellular localization of super35S::CHR00069684::GFP fusion protein in stem (E) and root (F). (e, f) Enlarged image of CHR00069684::GFP fusion protein in stem (e) and root (f). The red box area is the enlarged area. 


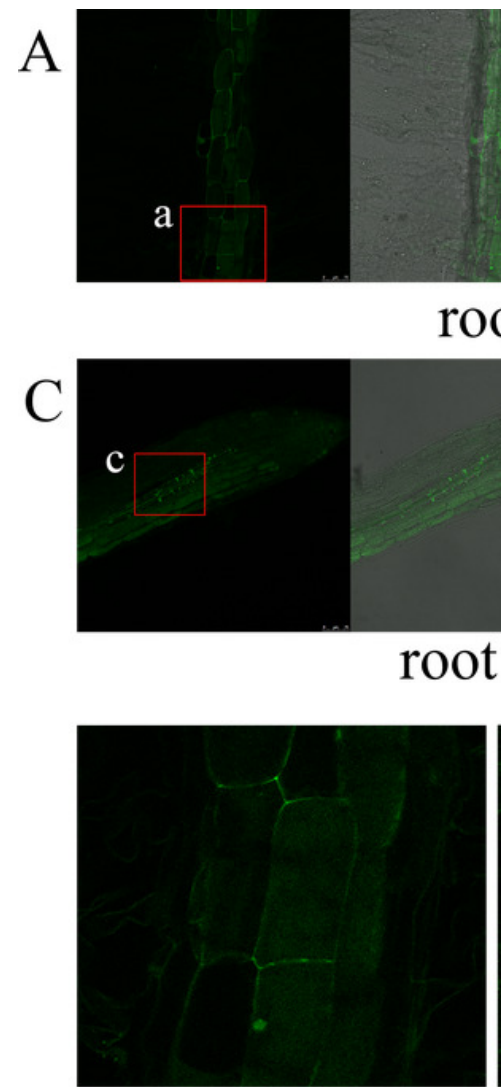

a

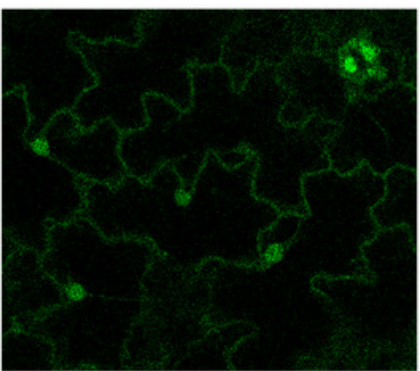

b

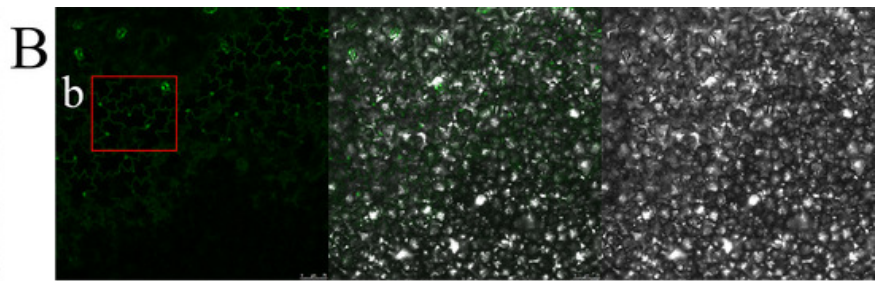

leaf

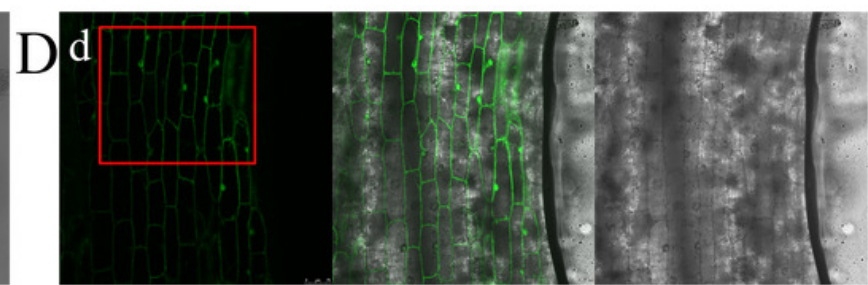

stem

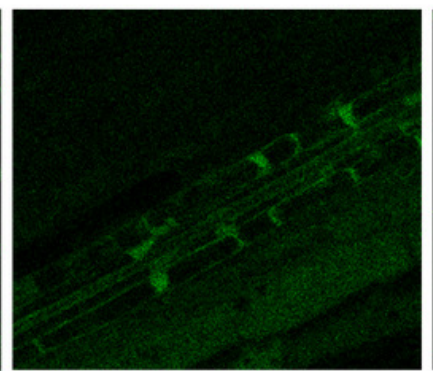

C

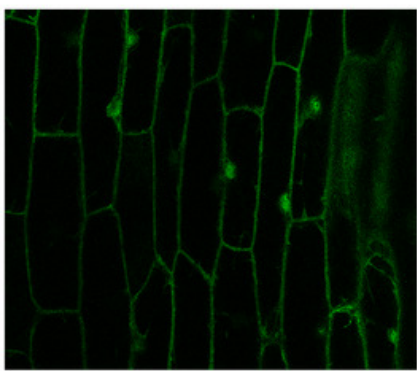

d

super35S::GFP

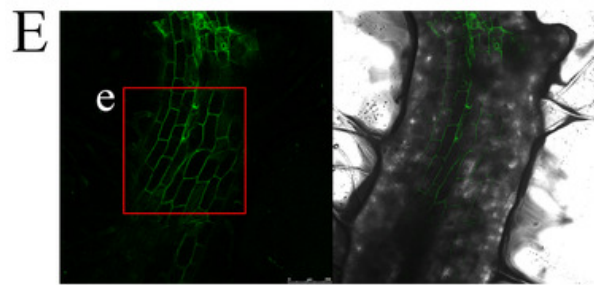

stem

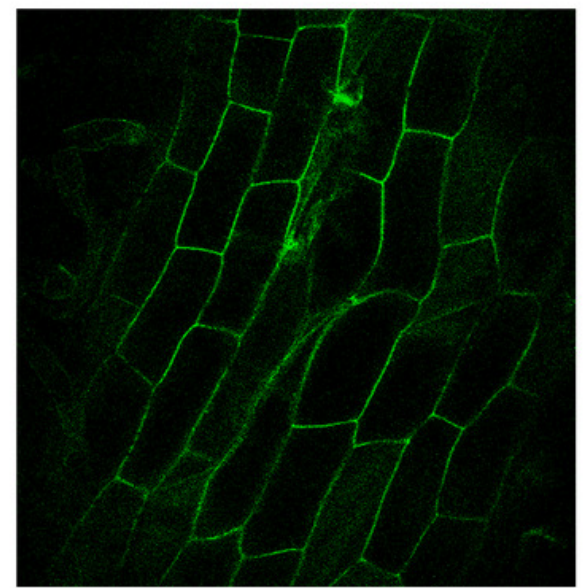

e

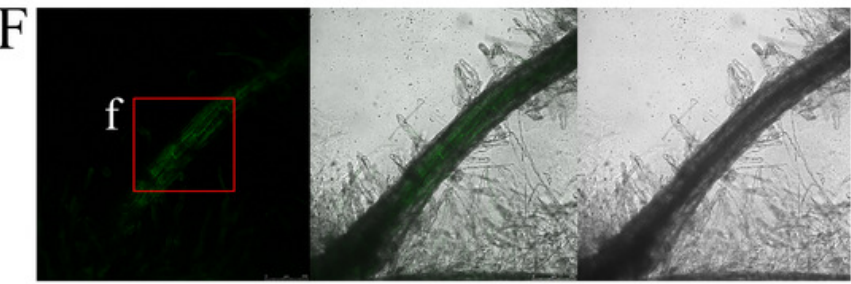

root

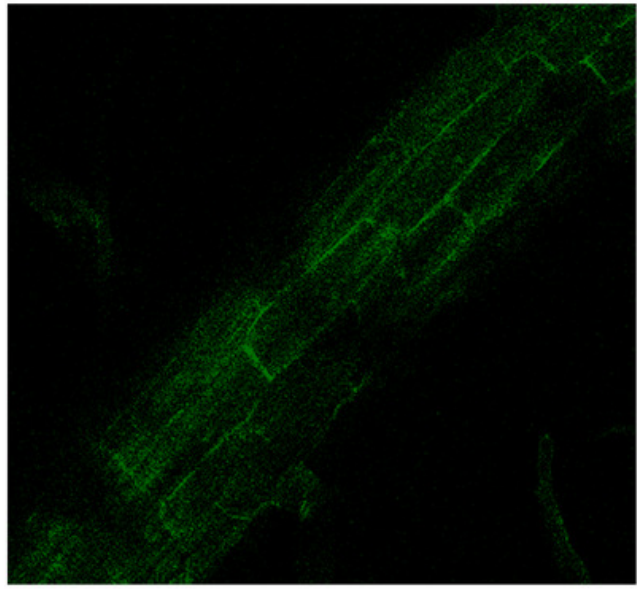

$\mathrm{f}$

super35S::CHR00069684::GFP 


\section{Figure 10}

The growth status of tobacco seedlings treated with $200 \mathrm{mmol} / \mathrm{L} \mathrm{NaCl}$ stress, $4^{\circ} \mathrm{C}$ low temperature stress and $200 \mu \mathrm{mol} / \mathrm{L}$ ABA treatment.

(A-C) Growth of super35S::CHR00069684::GFP transgenic tobacco seedlings under $\mathrm{NaCl}(\mathrm{A})$, ABA (B) and $4^{\circ} \mathrm{C}(\mathrm{C})$. (D-F) Growth of super35S::GFP transgenic tobacco seedlings under $\mathrm{NaCl}$ (D), $A B A(E)$ and $4^{\circ} \mathrm{C}(F)$. All treatments were grown in stress medium for 30 days.

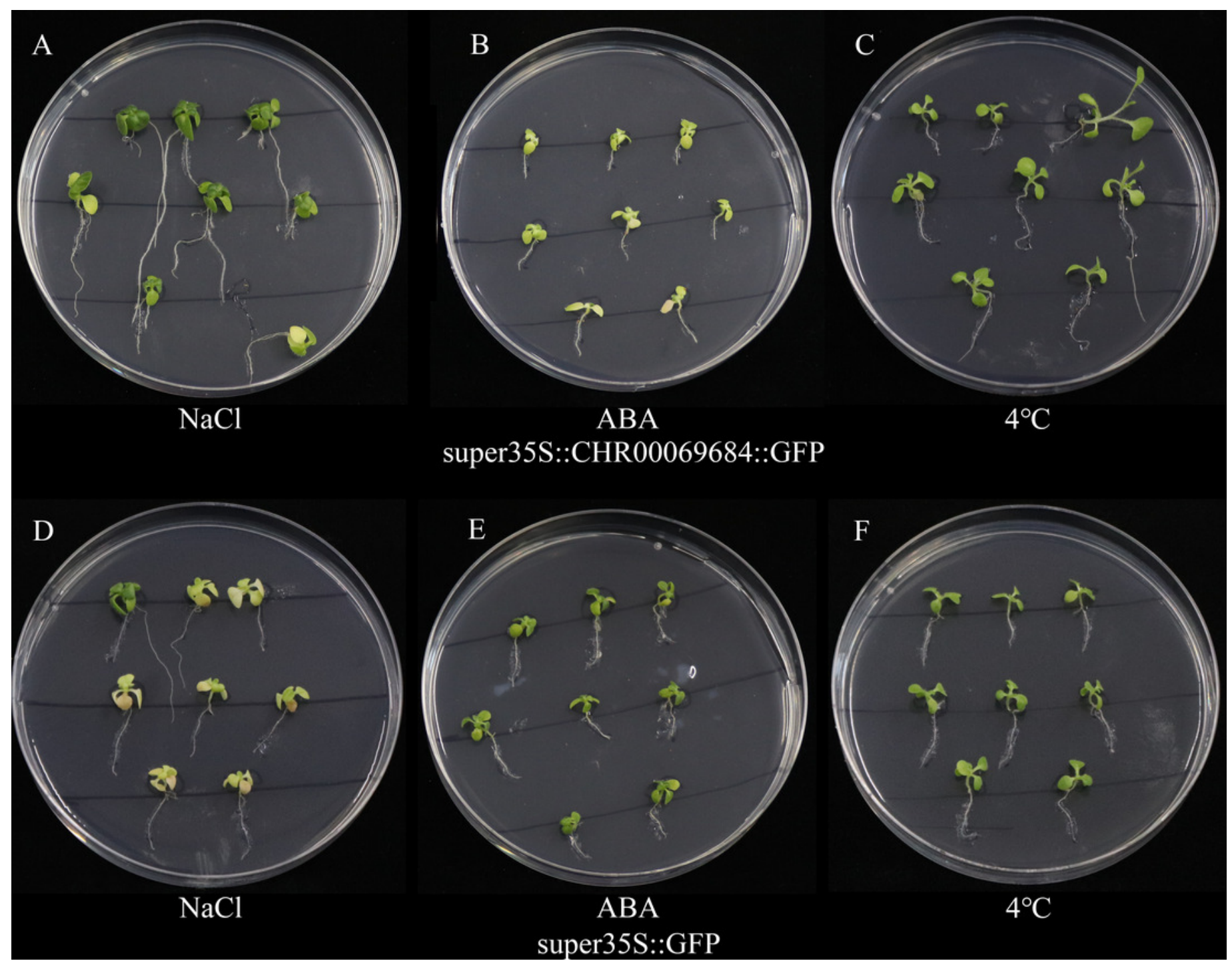




\section{Figure 11}

Growth and development of transgenic tobacco at 43 days.

(A) The growth and development status of super35S::CHR00069684::GFP transgenic tobacco.

(B) The growth and development status of super35S:: GFP transgenic tobacco. Both groups of tobacco grew in the same environment and underwent the same conservation management.

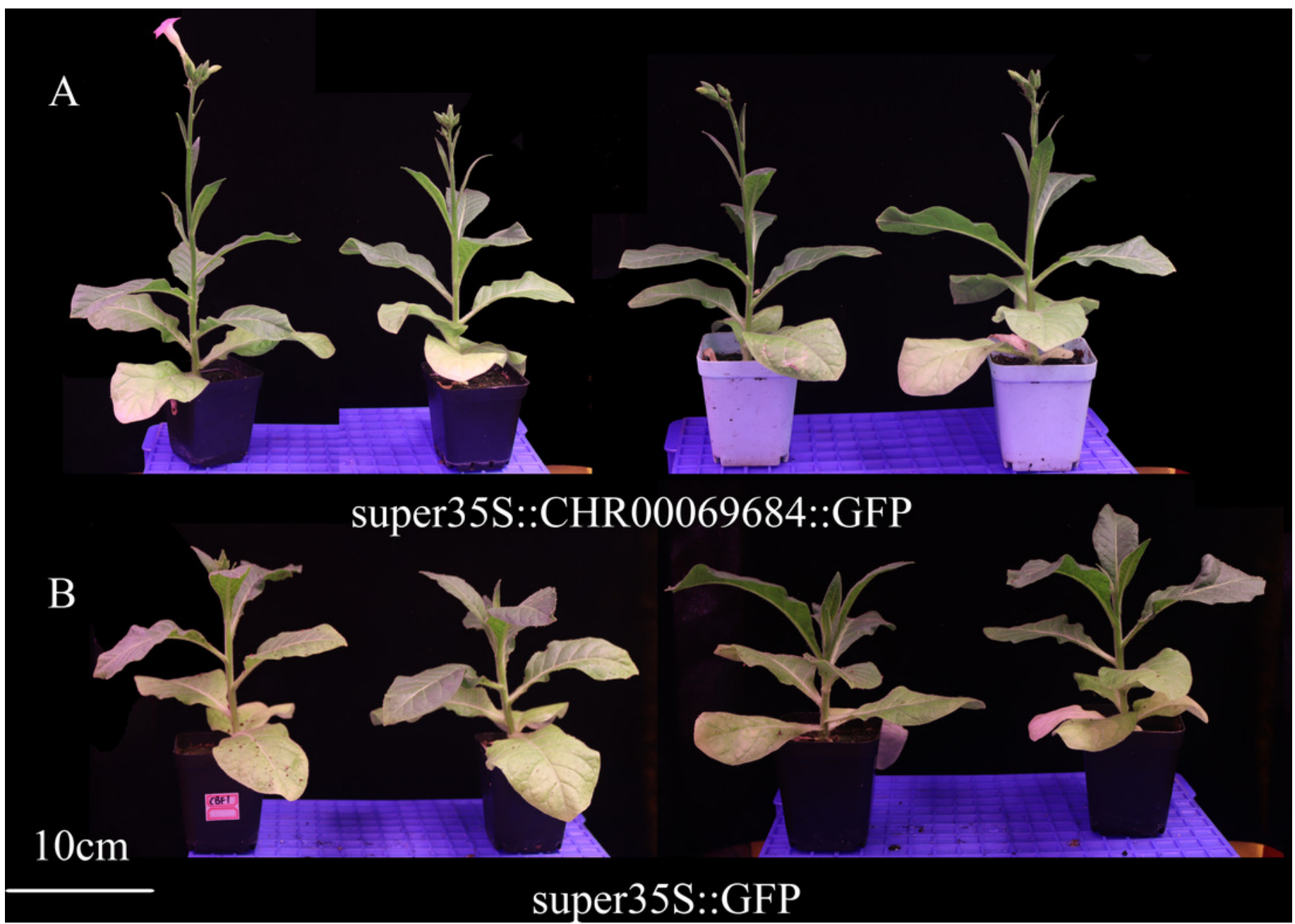


Figure 12

Sequence alignment and motif analysis of OsNAC7 subfamily genes.

Different color frames represent different motif regions. Different colored boxes show the positions of different motifs in genes.

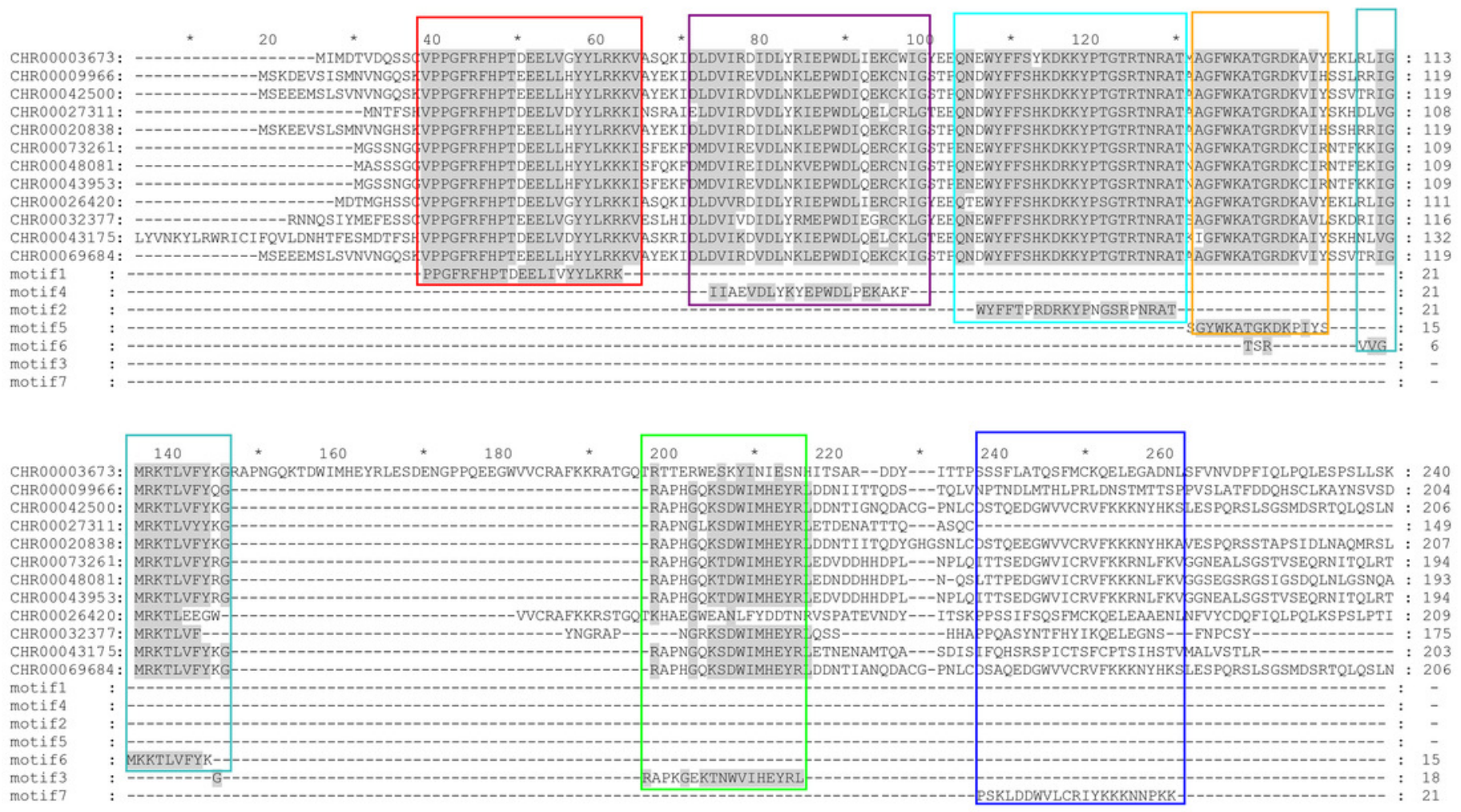




\section{Table 1 (on next page)}

RT-qPCR primers for the OsNAC7 subfamily genes of $C$. nankingense

Notes: actin7-F/R primer is an internal reference gene primer. 
Table 1 RT-qPCR primers for the OsNAC7 subfamily genes of C. lavandulifolium

\begin{tabular}{|c|c|c|c|c|}
\hline Number & Gene name & Primer sequence & GC CONTENT ( \%) & $\mathrm{TM}\left({ }^{\circ} \mathrm{C}\right)$ \\
\hline \multirow{2}{*}{1} & actin7-F & CGTTGCCCTGAGGTTCTCTT & 55 & 60.0 \\
\hline & actin7-R & СCTTGCTCATCCTGTCAGCA & 55 & 60.0 \\
\hline \multirow{2}{*}{2} & CHR00069684-F & CCAAGGCTCGATAGTCCCAC & 60 & 59.9 \\
\hline & CHR00069684-R & AGGAAGTCAGTTGCATGGGG & 55 & 60 \\
\hline \multirow{2}{*}{3} & CHR00042500-F & GAGCACCTCATGGCCAGAAA & 55 & 60.3 \\
\hline & CHR00042500-R & CACGACATACCACCCAACCA & 55 & 60 \\
\hline \multirow{2}{*}{4} & CHR00020838-F & AAAGGACGAGCACCTCATGG & 55 & 60 \\
\hline & CHR00020838-R & ACCСАССССТСТTCTTGAGT & 55 & 60.1 \\
\hline \multirow{2}{*}{5} & CHR00009966-F & CACCTCCCACGGCTTGATAA & 55 & 59.7 \\
\hline & CHR00009966-R & GTCAGCAAGTCACTCACGGA & 55 & 60 \\
\hline \multirow{2}{*}{6} & CHR00048081-F & ACTCCTGAAGATGGGTGGGT & 55 & 60.2 \\
\hline & CHR00048081-R & GCCTGCGAAAAAGAACCAGC & 55 & 60.7 \\
\hline \multirow{2}{*}{7} & CHR00043953-F & ATCTACCCGCCGAAGAGTCA & 55 & 60.4 \\
\hline & CHR00043953-R & GAGCTGCTTCAGAGGTGTCA & 55 & 59.7 \\
\hline \multirow{2}{*}{8} & CHR00073261-F & CCGACGAGGAACTGCTTCAT & 55 & 60.1 \\
\hline & CHR00073261-R & GGCGTCGACCCTATCTTACA & 55 & 59 \\
\hline \multirow{2}{*}{9} & CHR00032377-F & GGAAGGGACAAGGCAGTGTT & 55 & 60.2 \\
\hline & CHR00032377-R & CATGATGGGACGACTGGAGG & 60 & 59.9 \\
\hline \multirow{2}{*}{10} & CHR00026420-F & GTGGTGTGTCGTGCATTCAA & 55 & 59.3 \\
\hline & CHR00026420-R & ATTTACCTCTGTGGCTGGGC & 55 & 60 \\
\hline \multirow{2}{*}{11} & CHR00003673-F & ACGGGGAGAGATAAGGCAGT & 55 & 60 \\
\hline & CHR00003673-R & TCGGTCTTCTGTCCGTTTGG & 55 & 60 \\
\hline \multirow{2}{*}{12} & CHR00027311-F & AAGAGTTGTGCAGGCTTGGT & 55 & 60.1 \\
\hline & CHR00027311-R & GCTTTCCAAAATCCAGCTGCA & 47.6 & 60 \\
\hline \multirow{2}{*}{13} & CHR00043175-F & GCACCAACAGGGCTACAAAG & 55 & 59.4 \\
\hline & CHR00043175-R & CGTTAGGAGCTCGACCCTTG & 60 & 60.2 \\
\hline
\end{tabular}

2 Notes: actin7-F/R primer is an internal reference gene primer. 
Table 2 (on next page)

Research status of NAC family of rice and chrysanthemum 
1 Table 2 Research status of NAC family of rice and chrysanthemum

\begin{tabular}{|c|c|c|}
\hline Gene name & Subfamily & Function Annotation \\
\hline ONAC029/031 & OsNAC7 & Through alternative splicing, the thickening of fibrous cell walls during wood formation was regulates ${ }^{[78]}$. \\
\hline OsNAC106 & OsNAC7 & A negatively regulated NAC transcription factor. Osnac106 deletion mutants showed enhanced salt tolerance ${ }^{[48]}$. \\
\hline OsSND2 & OsNAC7 & The $A t S N D 2$ homologous gene. The overexpressed transgenic plants showed leaf curl, increased cellulose content ${ }^{79]}$. \\
\hline OsNAC5/ONAC071/AK102475 & ATAF & It can enhance the drought resistance, salt tolerance and cold tolerance of plants, and the phenotype is not affected ${ }^{[6]}$. \\
\hline OsNAC6/ONAC048/AK068392 & ATAF & $\begin{array}{l}\text { The homologous gene of } O s N A C 5 \text {. The overexpression of } O s N A C 6 \text { enhanced the tolerance of plants to high salt, drought and cold and its } \\
\text { overexpression can also reduce the range of disease and improve the resistance of rice blast }{ }^{[34,62-63]} \text {. }\end{array}$ \\
\hline OsNAC60 & NAC1 & OSNAC60 overexpression enhanced the resistance of transgenic plants to rice blast ${ }^{[66] .}$. \\
\hline OsNAC2/ONAC004/AK061745 & NAM & Negatively regulated $N A C$ TF. It can affect plant height and flowering time, reduce chlorophyll level, and accelerate leaf senescence ${ }^{[67-69]}$. \\
\hline ONAC45 & NAM & Under drought and high salt stress, the survival rate of plants with overexpression of this gene was higher than that of wild-type ${ }^{[70]}$. \\
\hline OsNAC10 & NAP & $\begin{array}{l}\text { The root epidermis, cortex and column cells of overexpressed rice plants increased significantly, and the water absorption capacity was } \\
\text { enhanced, which enhanced the drought and salt tolerance of the plants }{ }^{700} \text {. }\end{array}$ \\
\hline OsNAC041 & ONAC001 & Induced expression under salt stress. The rice Osnac041 mutant had higher plant height and stronger salt stress sensitivity [73]. \\
\hline OsNAC52 & ONAC001 & In A. thaliana overexpressed plants, transpiration of leaves decreased and drought resistance increased ${ }^{[74]}$ \\
\hline ONAC063 & ONAC022 & Improved salt tolerance of transgenic $A$. thaliana. Its overexpression related to salt stress were up-regulated ${ }^{[64]}$. \\
\hline OsNAC022 & ONAC022 & In overexpressed plants, the water loss rate and transpiration rate decreased, and drought and salt tolerance were enhanced ${ }^{[65]}$. \\
\hline OsNAC122/OsNAC131 & TERN & These two are homologous genes. In rice plants silenced by VIGS method, the ability to infect rice blast was enhanced ${ }^{[75]}$. \\
\hline OsNAP & NAP & Its overexpression could enhance the content of stress related genes, and improve the resistance of rice to different stress ${ }^{[72]}$. \\
\hline AaNAC1 & ATAF & Its overexpression can increase the content of artemisinin and improve resistance to drought and botrytis cinerea in $A$. апnиа ${ }^{[80]}$. \\
\hline DINAC1 & ONAC022 & It can involve in regulating plant tolerance to drought and salinity, and can improve high temperature tolerance of tobacco ${ }^{[59]}$. \\
\hline CINAC9 & SENU5 & $\begin{array}{l}\text { It can enhance the tolerance of transgenic } A \text { thaliana to salt, alkali and drought stress }{ }^{[76]} \text {. Its transgenic plants chrysanthemum niU9717 } \\
\text { can improve the resistance under salinization and drought stress }{ }^{[77]} \text {. }\end{array}$ \\
\hline
\end{tabular}

